\title{
An MHV macrodomain mutant predicted to lack ADP-ribose binding activity is severely attenuated, indicating multiple roles for the macrodomain in coronavirus replication
}

Lynden S. Voth ${ }^{\mathrm{a}, \mathrm{c}}$, Joseph J. O’Connor ${ }^{\mathrm{a}, \mathrm{c}}$, Catherine M. Kerr ${ }^{\mathrm{a}}$, Ethan Doerger ${ }^{\mathrm{a}}$, Nancy

5 Schwarting ${ }^{\mathrm{a}}$, Parker Sperstad ${ }^{\mathrm{a}}$, David K. Johnson ${ }^{\mathrm{b}}$, and Anthony R. Fehr ${ }^{\mathrm{a}, *}$

6 aDepartment of Molecular Biosciences, University of Kansas, Lawrence, Kansas 66045, USA

7 bolecular Graphics and Modeling Laboratory and the Computational Chemical Biology Core,

8 University of Kansas, Lawrence, Kansas 66047, USA

$9 \quad$ These authors contributed equally to this work 
ABSTRACT

All coronaviruses $(\mathrm{CoVs})$ contain a macrodomain, also termed Mac1, in non-structural

19 protein 3 (nsp3) which binds and hydrolyzes ADP-ribose covalently attached to proteins. Despite

20 several reports demonstrating that Mac1 is a prominent virulence factor, there is still a limited

21 understanding of its cellular roles during infection. Currently, most of the information regarding

22 the role of $\mathrm{CoV}$ Mac1 during infection is based on a single point mutant of a highly conserved

23 asparagine-to-alanine mutation, which is known to largely eliminate Mac1 ADP-

24 ribosylhydrolase activity. To determine if Mac1 ADP-ribose binding separately contributes to

25 CoV replication, we compared the replication of a murine hepatitis virus (MHV) Mac1 mutant

26 predicted to dramatically reduce ADP-ribose binding, D1329A, to the previously mentioned

27 asparagine mutant, N1347A. D1329A and N1347A both replicated poorly in bone-marrow

28 derived macrophages (BMDMs), were inhibited by PARP enzymes, and were highly attenuated

29 in vivo. However, D1329A was significantly more attenuated than N1347A in all cell lines tested

30 that were susceptible to MHV infection. In addition, D1329A retained some ability to block IFN-

$31 \beta$ transcript accumulation compared to N1347A, indicating that these two mutants impacted

32 distinct Mac1 functions. Mac1 mutants predicted to eliminate both binding and hydrolysis

33 activities were unrecoverable, suggesting that the combined activities of Mac1 may be essential

34 for MHV replication. We conclude that Mac1 has multiple roles in promoting the replication of

$35 \mathrm{MHV}$, and that these results provide further evidence that Mac1 could be a prominent target for 36 anti-CoV therapeutics. 


\section{IMPORTANCE}

38 In the wake of the COVID-19 epidemic, there has been a surge to better understand how

39 CoVs replicate, and to identify potential therapeutic targets that could mitigate disease caused by

40 SARS-CoV-2 and other prominent CoVs. The highly conserved macrodomain, also termed

$41 \mathrm{Mac1}$, is a small domain within non-structural protein 3. It has received significant attention as a

42 potential drug target as previous studies demonstrated that it is essential for CoV pathogenesis in

43 multiple animal models of infection. However, the various roles and functions of Mac1 during

44 infection remain largely unknown. Here, utilizing recombinant Mac1 mutant viruses, we have

45 determined that different biochemical functions of Mac1 have distinct roles in the replication of

$46 \mathrm{MHV}$, a model CoV. These results indicate that Mac1 is more important for CoV replication

47 than previously appreciated, and could help guide the development of inhibitory compounds that 48 target unique regions of this protein domain. 


\section{INTRODUCTION}

Coronaviruses $(\mathrm{CoVs})$ are of the family Coronaviridae in the Nidovirales order and are

52 responsible for a variety of diseases of both clinical and veterinary significance. These diseases

53 range from potentially lethal human respiratory diseases such as severe acute respiratory

54 syndrome (SARS)-CoV, SARS-CoV-2, and Middle East respiratory syndrome (MERS)-CoV; to

55 mammalian gastrointestinal diseases such as porcine epidemic diarrhea virus (PEDV) and avian

56 respiratory diseases such as infectious bronchitis virus (IBV) (1). There exists few vaccines or

57 broad-spectrum therapeutics to prevent and treat $\mathrm{CoV}$-induced disease. As SARS-CoV-2

continues to be a significant health threat, and as there will likely be further CoV outbreaks in the

59 future, there is an urgent need for a better understanding of the mechanisms used by CoVs to

60 promote their replication and cause severe disease.

Like other members of the Nidovirales order, $\mathrm{CoV}$ genetic information is stored as non-

62 segmented, positive-sense RNA ranging in size from $26-32 \mathrm{~kb}$, making them members of the

63 class of RNA viruses containing the largest genomes. These genomes can be broken down into

64 the region encoding structural and accessory proteins, comprising approximately $10 \mathrm{~kb}$ at the 3'

65 end of the genome, and the region encoding non-structural proteins (nsps), consisting of

66 approximately $20 \mathrm{~kb}$ at the 5 ' end of the genome. Conserved structural proteins include the

67 spike, envelope, membrane, and nucleocapsid proteins. Accessory proteins have important roles

68 in viral pathogenesis, such as antagonism of the type 1 IFN antiviral response, but are not

69 essential for in vitro viral replication. The nsps are translated into a long polyprotein and are

70 responsible for the virus' genomic and sub-genomic RNA synthesis. The nsps perform a variety

71 of functions, and include the polymerase, helicase, 2 proteases, and many others. However, the

72 function of many nsps are still being fully determined or remain completely unknown. The 
73 largest nonstructural protein, nsp3, contains several modular domains including a ubiquitin-like

74 domain, an acidic domain, one or two papain-like protease (PLP) domains, multiple

75 transmembrane domains, and one or more macrodomains $(1,2)$.

76 Macrodomains are globular protein domains present in many different life forms,

77 including humans, yeast, bacteria, and several families of positive-sense RNA viruses. They have

78 a highly conserved "sandwich" structure that includes several central $\beta$-sheets surrounded by $3 \alpha$ -

79 helices on each side $(3,4)$. The primary biochemical functions of macrodomains are to bind and

80 hydrolyze ADP-ribose from proteins. There are additional macrodomains (Mac2/Mac3) in some

81 CoVs, including SARS-CoV-2, that do not bind ADP-ribose and instead bind to nucleic acids or

82 cellular proteins to promote virus replication (5-11).

83 ADP-ribosylation is the posttranslational covalent addition of ADP-ribose to proteins by

84 ADP-ribosyltransferases that utilize $\mathrm{NAD}^{+}$as a substrate. ADP-ribose can be added to proteins

85 as single subunits (mono-ADP-ribosylation or MAR) or as chains of multiple subunits (poly-

86 ADP-ribosylation or PAR). This process is performed intracellularly by poly-ADP-ribose

87 polymerases (PARPs), also known as diphtheria toxin-like ADP-ribosyltransferases (ARTDs).

88 There are 17 human PARPs with PARP-1 being the most well-studied, as it mediates most of the

89 PARylation that occurs in the cell. Much less is known about the MARylating PARPs, though

90 many of them are interferon stimulated genes (ISGs) and some have demonstrated anti-viral

91 activities (12). For example, PARP12-mediated ADP-ribosylation impedes the replication of

92 Zika virus by ADP-ribosylating NS1 and NS3, leading to their proteasomal degradation (13).

93 PARP activity is countered by several dePARylating or deMARylating enzymes, including

94 PARGs (polyADP-ribose glycohydrolase), ARHs (ADP-ribosylhydrolases), and macrodomains

$95 \quad$ (14). 
Recombinant viruses mutated at a highly conserved asparagine residue in the primary

$97 \mathrm{CoV}$ macrodomain (herein referred to as Mac1) have been engineered for multiple CoVs to

98 understand the role of Mac1 during infection. This residue was targeted because its mutation to

99 alanine had eliminated Mac1 phosphatase activity, was later shown to eliminate ADP-

100 ribosylhydrolase activity, and is completely conserved amongst all enzymatically active

101 macrodomains (15-18). Structurally, it is positioned to provide critical hydrogen bonds with the

102 terminal ribose, positioning the ADP-ribose for hydrolysis $(15,19,20)$. Recombinant viruses

103 with this mutation generally replicate normally in tissue culture cells, but are highly attenuated in

104 vivo $(16,21-23)$. These reports have established Mac1 as a prominent virulence factor and

105 potential therapeutic target (4). For instance, the SARS-CoV Mac1 mutant virus, N1040A,

106 replicates poorly in mice and induces an increased IFN and pro-inflammatory cytokine response

107 in mice and Calu-3 cells, a bronchial epithelial cell line. (16). These results demonstrated that

108 Mac1 is required for the ability of SARS-CoV to fully inhibit IFN and cytokine induction.

109 Similar results were observed following infection of bone-marrow derived macrophages

110 (BMDMs) with the JHM strain of MHV (JHMV) containing the same asparagine-to-alanine

111 mutation (N1347A) in Mac1 $(19,23)$. We also found that treatment with PARP inhibitors or

112 siRNAs targeting PARP12 or PARP14 enhanced the replication of N1347A. These results

113 demonstrated that the CoV nsp3 Mac1 domain is required to prevent PARP-mediated inhibition

114 of virus replication (23).

115 While these studies have provided significant insight into the role of the CoV Mac1

116 domain, they are largely based on a single asparagine-to-alanine mutation. As this mutation may

117 more significantly impact enzymatic activity than ADP-ribose binding, it remains unclear

118 whether the ADP-ribose binding ability of Mac1 may have additional roles in CoV replication. 
119 ADP-ribose can be covalently bound to a number of different amino acids, including acidic,

120 basic, serine, and cysteine residues, while macrodomains have only been shown to hydrolyze

121 ADP-ribose attached to acidic residues. Previous studies on the alphavirus macrodomains

122 showed that mutations separating the ADP-ribose binding and hydrolase activities result in

123 distinct phenotypes during virus infection $(24,25)$. These results indicate that macrodomain

124 binding to ADP-ribosylated proteins with ADP-ribose attached at non-cleavable residues, such as 125 serine, may have functions distinct from its ADP-ribosylhydrolase activity.

126 Here, we created recombinant JHMV with two distinct mutations that are predicted to

127 impact Mac1 ADP-ribose binding. We found that a D1329A mutant, predicted to abrogate ADP-

128 ribose binding, had more severe replication defects in cell culture than N1347A but mostly

129 retained its ability to block IFN production when compared to N1347A. PARP inhibitors

130 enhanced the replication of D1329A, and NAD enhancing compounds further decreased its

131 replication, indicating that the defects of this virus was due to PARP activity. Finally, we failed

132 to recover a recombinant virus containing both the D1329A and N1437A mutants, or a separate

133 mutant predicted to have diminished ADP-ribose binding and hydrolysis activity, G1439V,

134 suggesting that the combined activities of Mac1 are essential for JHMV replication. 


\section{RESULTS} structure for the MHV Mac1, we used computer modeling to predict its overall structure and the structure of its ADP-ribose binding pocket (Fig. 1A-B). Not surprisingly, the structure of the MHV Mac1 protein is similar to other CoV Mac1 proteins (Fig. 1C-D). One residue predicted to

141 impact ADP-ribose binding is a highly conserved aspartic acid residue located in a loop region

142 between $\beta 2$ and $\alpha 1$ of MHV Mac1 (D22) (Fig. 1A-B, E-F). This residue is known to either make

143 a critical hydrogen bond with the N6 nitrogen of the adenine ring or mediate water contacts with

144 this molecule (Fig. 1B) $(15,20,26,27)$. Its position in the ADP-ribose binding pocket is well

145 conserved and largely superimposes with that of the SARS-CoV-2 and MERS-CoV Mac1

146 proteins (Fig. 1E-F). Mutation of this aspartic acid in multiple macrodomains virtually eliminates

147 ADP-ribose binding, but maintains some ADP-ribosylhydrolase activity $(16,28,29)$. Therefore,

148 determining the role of this residue in virus replication and pathogenesis may provide unique

149 insight into the functions of Mac1. Another residue of interest is an asparagine located in a loop

150 between $\beta 7$ and $\alpha 6$ of MHV Mac1 (N156) (Fig. 1A-B, E-F). This residue is mostly conserved

151 amongst $\beta$-CoVs, and in our MHV-1 modeled structure it appears to provide hydrogen bonds to

152 the proximal ribose. It has also been proposed to provide similar interactions in the SARS-CoV

153 and MERS-CoV Mac1 proteins $(20,26)$. This residue is often found as a hydrophobic amino

154 acid in other CoV and viral macrodomains, including phenylalanine in SARS-CoV-2 Mac1 (30).

155 These side chains appear to be in close contact with the adenine base and may stack against the

156 adenine ring to create water-mediated hydrogen bonds with the proximal ribose (31). Based on

157 these observations, we hypothesized that alanine mutations at these residues would provide

158 further insight into the role of Mac1 ADP-ribose binding in CoV replication and pathogenesis. 
160 N1465A replicates like WT virus. To test the role of the D22 (D1329 in pp1a) and N156

161 (N1465 in ppla) residues in the context of MHV replication, we first engineered recombinant

162 JHMV BACs containing the D1329A and N1465A mutations using a two-step Red

163 recombination with the endonuclease I-SceI as previously described $(22,32)$. These recombinant

164 viruses, termed D1329A and N1465A, were easily recovered and replicated in cell culture.

165 Following reconstitution of virus, we sequenced the Mac1 region and confirmed that these

166 mutations had been retained following passaging.

We first compared the replication of D1329A and N1465A with that of WT and N1347A

168 viruses in BMDMs. We previously showed that N1347A had significantly decreased replication

169 in BMDMs, and initially hypothesized that D1329A and N1465A would be similarly affected.

170 While WT and N1465A viruses were able to replicate to similar levels at all time points,

171 N1347A and D1329A were highly attenuated, showing a greater than 1-log replication defect

172 throughout the infection (Fig. 2A). We next tested the replication of D1329A and N1465A on

173 17Cl-1 fibroblasts, a common cell line that is highly permissive for MHV replication. As

174 expected, N1347A replicated like WT virus in these cells, as did N1465A, but surprisingly

175 D1329A replicated poorly in these cells with a replication defect of $\sim 1-\log$ (Fig. 2 B-C) and

176 produced reduced levels of viral nucleocapsid $(\mathrm{N})$ and spike $(\mathrm{S})$ protein (Fig. 2D). To confirm

177 that this defect was not due to a second-site mutation in the BAC, we repaired this mutation to

178 create the BAC clone, repD1329. The virus recovered from this BAC clone replicated well and

179 produced viral proteins at WT virus levels in 17Cl-1 cells, confirming that the replication defect

180 was due to mutation of D1329 (Fig. 2C-D). 
Next, we tested whether D1329A was defective in additional MHV susceptible cells. In both L929 fibroblasts and DBTs (delayed brain tumor), an astrocytoma cell line, D1329A was highly attenuated with replication defects of approximately 2-logs, and very little $\mathrm{N}$ and $\mathrm{S}$ protein accumulated in both cell types compared to WT virus (Fig. 2E-H). Interestingly, we found that N1347A also replicated poorly and produced reduced N and S protein in DBT cells, though it replicated better than D1329A (Fig. 2G-H).

The MHV Mac1 D1329A virus mostly retains the ability to repress IFN production

in BMDMs. The dramatic reduction in replication of D1329A could indicate that it had lost all ADP-ribose binding and hydrolase activity, while N1347A maintains at least partial activity for one or both of these functions. Alternatively, D1329A may primarily impact ADP-ribose binding

191 while N1347A primarily affects Mac1 enzymatic activity. Current biochemical data based on 192 analyses of similar macrodomains favor the latter hypothesis. Previous reports have found that

193 the aspartic acid mutation leads to complete or nearly complete loss of binding but these mutants 194 retained some hydrolase activity. In contrast, the asparagine mutation leads to the near complete 195 loss of Mac1 hydrolysis activity but had only a 2 to 3-fold reduction in ADP-ribose binding (16, $19625,28,29,33)$.

To further test these opposing hypotheses, we measured the level of IFN- $\beta$ mRNA at 6 198 and 12 hpi in BMDMs infected with WT, N1347A, D1329A, and N1465A. We previously found 199 that N1347A infection led to a $>1-\log$ increase in IFN- $\beta$ transcript levels in BMDMs (23). We 200 predicted that if D1329A had less overall activity compared to N1347A, its infection would lead 201 to an equivalent or greater increase in IFN- $\beta$ transcript levels than N1347A. However, we might 202 expect the D1329A mutant infected cells to have reduced IFN- $\beta$ transcript levels compared to 203 N1347A infected cells if hydrolysis was primarily responsible for blocking IFN production. 
204 Here, infection with N1347A resulted in a $>19$-fold increase in IFN- $\beta$ mRNA levels at 12 hpi and a smaller difference at 6 hpi (Fig. 3A), consistent with our previous report. N1465A-infected cells had similar IFN- $\beta$ transcript levels to those infected with WT virus, further indicating that this mutation does not affect Mac1 function. However, instead of observing further increases of IFN- $\beta$ mRNA following D1329A infection, we found that IFN- $\beta$ mRNA levels in BMDMs were reduced 3.5-fold when compared to N1347A, despite having roughly the same level of viral genomic RNA (gRNA) in the cells in this specific experiment (Fig. 3A-B). These results indicate

211 that D1329A retains some ability to block IFN- $\beta$ transcription, and thus likely has more, and not

212 less, ADP-ribosylhydrolase activity than N1347A. These results, in combination with results in

213 Fig. 2, suggests that Mac1 utilizes multiple mechanisms to promote virus replication and block

214 innate immune responses in cell culture.

216 boosting the PARP substrate $\mathrm{NAD}^{+}$with nicotinamide riboside further inhibits D1329A

217 replication. The D1329 residue is known to be critical for ADP-ribose binding from multiple

218 studies $(28,29)$, however it is also conceivable that this mutation could affect other functions of

219 the CoV macrodomain, such as Papain-Like Protease (PLPro) binding (34). To address this

220 possibility, we treated cells with inhibitors that target host PARP enzymes. If the ADP-

221 ribosyltransferase activity of PARP enzymes are restricting the replication of D1329A, we would

222 expect to see at least partial restoration of virus replication in the presence of PARP inhibitors, as

223 we previously showed with N1347A (23). First, we performed MTT assays to confirm that the

224 PARP inhibitors XAV-939 and Olaparib (2281) did not affect the metabolic capacity of BMDMs

225 or 17Cl-1 cells. BMDM and 17Cl-1 cells were treated with XAV-939 and 2281 for 24 hrs and

226 MTT levels were measured. Neither compound resulted in notable metabolic changes at the 
227 working concentration of $10 \mu \mathrm{M}$ (Fig. 4A-B). In virus replication experiments, both compounds

228 significantly enhanced the replication of D1329A in BMDMs and in 17Cl-1 cells, by

229 approximately 5 and 3-fold, respectively, but had no effect on WT virus (Fig. 4C-D). In addition,

230 the level of enhanced replication in BMDMs for D1329A was similar to that of N1347A. These

231 results indicate that PARP-mediated ADP-ribosylation inhibits the replication of D1329A.

232 To provide further evidence for PARP-mediated inhibition of D1329A, we hypothesized

233 that increasing PARP activity using nicotinamide riboside chloride (NR) would lead to further

234 reduction in D1329A replication. NR enhances PARP activity by increasing the intracellular

235 levels of the PARP substrate, $\mathrm{NAD}^{+}(35,36)$. We recently showed that NAD levels are depleted

236 following MHV infection, and that restoring NAD levels with NR and other NAD boosting

237 compounds both increased PARP activity and decreased the replication of N1347A (37). We first

238 confirmed that NR did not significantly decrease the metabolic activity of BMDM or 17Cl-1

239 cells at and above the working concentration of $100 \mu \mathrm{M}$ (Fig. 5A-B). Rather than decreasing

240 metabolic activity, 17Cl-1 cells treated with NR seemed to have slightly increased metabolic

241 activity, though this was not statistically significant (Fig. 5B). For the infection, we pretreated

242 these cells with NR for 4 hrs, then infected them with WT and D1329A, added fresh NR after the

243 infection and then collected both cell-free and cell-associated virus at 20 hpi. Similar to our

244 results with N1347A, we found that NR significantly reduced the replication of D1329A virus in

245 both cell types by $>5$-fold, but had no impact on WT virus (Fig. 5C-D). These results indicate

246 that NR enhanced PARP activity that further reduced D1329A virus replication, but was

247 countered by Mac1 in WT virus infected cells.

249 culture may not mimic what happens in vivo, we tested the effect of these mutations in mice, 
250 where WT JHMV causes a lethal encephalitis $(22,38)$. We infected C57BL/6 mice with

251 recombinant WT, N1347A, and N1465A JHMV at $3 \times 10^{4}$ PFU and monitored weight loss as a

252 clinical sign of disease progression (Fig. 6A-B). N1347A was included as an attenuated control

253 virus as it was previously shown to cause minimal disease in B6 mice (22). We hypothesized that

254 N1465A would cause disease similar to WT virus, as all cell culture experiments indicated that

255 this mutation had not affected Mac1 function. Indeed, N1465A infected mice lost weight and

256 were euthanized due to severe disease at nearly the same rate as WT infected mice, while

257 N1347A did not cause any weight loss and all mice survived its infection (Fig. 6A-B). These

258 results provide further evidence that mutation of N1465 does not significantly affect the function

259 of Mac1.

260 Next, we infected mice with $3 \times 10^{3}$ PFU of WT and D1329A virus. This low dose was

261 used because titers of the D1329A virus stocks were significantly lower than other viruses.

262 Regardless, WT virus still caused significant weight loss and the infected mice succumbed to this

263 infection at roughly the same rate as did those receiving the higher dose of virus. Interestingly,

264 the D1329A virus did lead to weight loss and lethality in 5 out of 7 male mice and 1 out of 6

265 female mice (Fig. 6C-D). Since this result was unexpected, we collected the brains of 1 female

266 and 2 male mice that succumbed to infection with D1329A and sequenced the Mac1 region of

267 the MHV genome. We found that in 2 of the 3 mice, the alanine reverted back to aspartic acid,

268 while in the third mouse there were 2 second-site mutations in the N-terminus of the

269 macrodomain, L1313V and K1327E (Fig. 6E). As all mice sequenced had reverted virus, it

270 appears that D1329A replicates poorly in vivo and is especially prone to reversion. We conclude

271 that D1329A is highly attenuated in vivo. 


\section{Recombinant JHMV with mutations predicted to impact both ADP-ribose binding}

and hydrolysis are not recoverable. To further test the hypothesis that Mac1 has multiple roles

274 in virus replication, we created a D1329A/N1347A double mutant BAC clone. We hypothesized

275 that if the D1329 residue confers a unique role in virus replication when compared to N1347,

276 then a double-mutant virus may be even more attenuated than either single mutant. Consistent

277 with this hypothesis, we were unable to recover the D1329A/N1347A virus following 12

278 transfection attempts from 2 separate BAC clones. Each of these experiments included

279 successful transfections of other BACs as positive controls (Table 1).

Introducing multiple mutations into the macrodomain could disrupt its structure and thus

281 the inability to recover this virus could be due to impairment of additional nsp3 functions outside

282 of Mac1. To provide further evidence that the loss of Mac1 function is lethal for JHMV, we

283 engineered an additional mutant, G1439V into our JHMV BAC clone. This mutation has

284 previously been introduced into SARS-CoV and SARS-CoV-2 recombinant Mac1 proteins, and

285 both proteins had minimal, if any, ADP-ribosylhydrolase activity $(16,39)$. Furthermore,

286 computational modeling of an ADP-ribose bound structure of the SARS-CoV and MERS-CoV

287 Mac1 proteins found that ADP-ribose binding was highly unfavorable when introducing the G-V

288 mutation, with a ddG of binding of $\sim 9$ and 10 rosetta energy units (REUs), respectively. After

289 transfection, we only recovered this virus $4 / 11$ times for a $37 \%$ recovery rate (Table 1 ). Upon

290 sequencing the recovered viruses, we found that most had reverted to WT virus. However, one

291 clone instead had evolved a second site mutation of alanine to threonine in the residue

292 immediately upstream of V1439. To determine if this mutation was responsible for the ability of

293 this virus to replicate, we created a A1438T/G1439V mutant BAC clone. We were able to easily

294 recover this virus, indicating that this mutation was the reverting mutation that allowed the 
295 outgrowth of one of the G1439V viruses (Table 1). A1438T/G1439V replicated better than

296 D1329A on L929 cells, but had a 3-fold replication defect and produced less viral protein on

297 L929 cells compared to WT virus (Fig. 7A-B). It also did not cause severe disease in mice,

298 indicating this mutation only partially recovered Mac1 functions (Fig. 7C-D). In total, we have

299 created two separate Mac1 recombinant JHMV clones (D1329A/N1347V and G1439V) that

300 were not recoverable without second site or reverting mutations. These results suggest that the

301 combined ADP-ribose binding and hydrolysis activities of Mac1 are essential for JHMV

302 replication.

\section{DISCUSSION}

Here we show that the JHMV Mac1 protein domain promotes virus replication in multiple ways, likely by utilizing both its ADP-ribose binding and ADP-ribosylhyrolase activities. Based on prior reports demonstrating the importance of Macl for CoV pathogenesis, there has been particular interest in developing therapeutic strategies targeting the SARS-CoV-2 Mac1 domain. (16, 31, 39-44). These results further demonstrate the importance of Mac1 for

310 CoV replication and could have implications in the design of compounds targeting the ADP-

311 ribose binding domain of Mac1.

312 Previous studies of the alphavirus macrodomain has provided unique insight into the 313 separate binding and enzymatic activities of these protein domains $(24,25,29,45)$. Chikungunya

314 (CHIKV) and Sindbis virus mutations that impact these activities are highly attenuated in cell 315 culture and in vivo. In fact, CHIKV macrodomain mutations that reduced hydrolysis activity by 316 as little as $25 \%$ are attenuated, and any mutation that eliminated binding activity was 317 unrecoverable (29). In addition, two mutants were further analyzed for their impact on the virus 
318 replication cycle, G32S (reduced hydrolysis and binding) and Y114A (decreased hydrolysis but

319 increased binding). While both mutants had reduced virus replication as measured by plaque

320 assay, the G32S mutant had greatly reduced levels of viral RNA and protein production early in

321 the infection, while Y114A had normal levels of viral RNA and protein until later stages of

322 infection. It was concluded that a CHIKV with ADP-ribose binding defects is unable to properly

323 initiate replication, while a hydrolase deficiency negatively affects the later stages of virus

324 replication.

To determine if there may be additional phenotypes associated with the ADP-ribose

326 binding ability of the CoV Mac1, we chose to mutate residues in the adenine binding pocket.

327 First, we targeted N1465, located in a loop between $\beta 7$ and $\alpha 6$, which appears to provide a

328 hydrogen bond with the proximal ribose (Fig. 1B). Despite its apparent ability to provide

329 interactions with the proximal ribose $(20,26)$, we found that mutation of this residue to alanine

330 had no impact on JHMV replication in any cell type we tested (Fig. 2), and also did not affect the

331 ability of JHMV to cause disease in mice (Fig. 6A-B). From these results we conclude that

332 N1465 does not contribute significantly to the ability of the JHMV Mac1 to promote replication

333 or cause disease.

334 Next, we targeted a conserved aspartic acid that makes a critical hydrogen bond with the

335 N7 nitrogen of the adenine base, D1329. While D1329 behaved similarly to N1347A in

336 BMDMs, it was substantially attenuated in multiple cell lines, including 17Cl-1, L929, and DBT

337 cells. Importantly N1347A replicates normally, or with only modest defects in these cells,

338 suggesting that D1329 contributes to virus replication in a unique manner compared to N1347.

339 This is consistent with biochemical data from other macrodomains that demonstrates that the D-

340 A mutation leads to dramatic loss of ADP-ribose binding, while the asparagine mutation only 
341 results in a 2-3-fold loss of ADP-ribose binding $(25,28,29)$. In mice we found that D1329A

342 often reverted. While in some cases it had reverted to WT, in one case two novel mutations near

343 D1329, L1313V and K1327E appeared. Both residues are found outside of the position of the

344 aspartic acid though not in near contact with the substrate (data not shown), making it unclear if

345 and how either of these amino acid changes helped enhance the replication of D1329A in vivo.

346 However, it is possible that the D1329A mutation could have significantly affected the structure

347 in this region of Mac1, causing a rearrangement of these residues. It will be of interest to

348 determine if one or both of these mutations can help restore ADP-ribose binding activity and

349 virus replication of this mutant.

350 In addition, we have shown that the defect of D1329 is due to PARP-dependent ADP-

351 ribosylation and not an additional Mac1 function. PARP inhibitors increased D1329A replication

352 in 17Cl-1 and BMDMs (Fig. 4), and NR, which increases levels of the PARP substrate NAD ,

353 further decreased its replication in these cell types (Fig. 5) (35-37). From these results it is likely

354 that ADP-ribose binding is a critical component of MHV replication. In contrast, D1329A did

355 not induce IFN $\beta$ transcript levels to the same degree as N1347A, which indicates that this virus

356 retains some function that is lost with N1347A. A logical explanation is that ADP-

357 ribosylhydrolase activity is responsible for Mac1 inhibition of IFN $\beta$ transcript levels, and that

358 N1347A has lost most, if not all of this enzyme activity while D1329A retains at least some

359 hydrolase activity. This is consistent with biochemical data of other CoV Mac1 mutant proteins

360 showing that the N-A mutation largely ablates hydrolase activity while the D-A mutant retains

361 some hydrolase activity $(15,16,18,25,29)$.

362 Interestingly, we were unable to recover a double mutant virus (D1329A/N1347A) or a

363 separate mutant designed to negatively impact both binding and hydrolase activities (G1439V). 
364 These results indicate that the combined activities of Mac1 may be essential for JHMV

365 replication. Intriguingly, we identified a second site mutation for G1439V in an immediately

366 adjacent residue, A1438T, that allowed it to replicate. Of note, all alphaviruses have a threonine

367 in this position. Based on computer modeling, we predict that this mutation may affect the

368 network of water molecules in the vicinity of these residues and could potentially improve either

369 the ADP-ribose binding or hydrolysis activity of Mac1 (Fig. 7C-D). After recreating an isogenic

370 BAC clone with these two mutations, A1438T/G1439V, we found that this clone was easily

371 recoverable and replicated in cell culture, though it had a mild replication defect compared to

372 WT virus in L929 cells and was attenuated in vivo. Regardless, these results confirmed that this

373 mutation allowed us to recover the G1439V mutant virus and provided even more evidence that

374 Mac1 is a potential therapeutic target for CoVs. Mac1 could be targeted by: i) using inhibitors

375 specific to the macrodomain; ii) enhancing PARP activity by increasing $\mathrm{NAD}^{+}$levels by

376 consumption of NR; or iii) a combination of both.

377 The primary challenge remaining is to identify targets of Mac1 that contribute to the

378 inhibition of Mac1 mutant viruses. Macrodomain proteins have only been demonstrated to

379 remove ADP-ribose from acidic residues (4), and several PARPs that are induced by virus

380 infection ADP-ribosylate acidic residues $(29,46)$. But recent proteomic analyses have shown that

381 in certain conditions, ADP-ribosylation of acidic residues is rare or even absent (47). Thus, it's

382 likely that viral macrodomains would also target proteins modified at non-acidic residues such as

383 serine, cysteine, or lysine, even if they cannot hydrolyze these ADP-ribose modifications. Mac1

384 binding to this modification may dramatically impact the biochemical activities of its target

385 proteins, for instance, by altering protein-protein interactions. While Mac1 is likely to target both

386 cellular and viral proteins during infection, the severe and universal defect of D1329A indicates 
387 that its binding activity may target an ADP-ribosylated CoV protein. We recently found that the

$388 \mathrm{CoV}$ nucleocapsid $(\mathrm{N})$ protein is ADP-ribosylated. Interestingly, this modification was

389 unchanged following infection with N1347A, indicating it is not cleaved by the macrodomain

390 but may be a target for macrodomain binding (48). Regardless, further investigation into how

391 PARP-mediated ADP-ribosylation impacts the function of viral and cellular proteins is likely to

392 uncover unique insights into the replication of CoVs.

393 
MATERIALS AND METHODS

Molecular modeling of the MHV Mac1 protein. A model of MHV Mac1 protein bound to ADP-ribose was generated using RosettaCM (49), using the ADP-ribose bound MERS-CoV Mac1 structure, 5DUS, as a template (20). Following the threading step, the pose of ADP-ribose bound to the MERS-CoV macrodomain was included in the refinement process to create a model of the MHV Mac1 protein bound to ADP-ribose. The top scoring model was aligned to the MERS Mac1 protein, and three structural waters that participate in hydrogen bond networks bridging the protein to ADP-ribose (water residues 312, 365, and 384) were added to the MHV model, followed by hydrogen bond optimization and minimization within Maestro by Schrodinger.

Rosetta Cartesian ddG calculations. The structures of the MERS-CoV and SARS-CoV Mac1 proteins bound to ADP-ribose, 5DUS (20) and 2FAV (15) respectively, were stripped of all waters but the three structural waters bridging the macrodomain to ADP-ribose (20). These structures, as well as the model of the MHV bound to ADP-ribose and the three waters, were prepared for $\mathrm{ddG}$ prediction by relaxing into cartesian space using coordinate restraints via Rosetta (50). The lowest energy structure was selected for ddG prediction of the G1439V mutant using Rosetta's cartesian ddG protocol (51). By default, cartesian ddG protocol runs for three

411 iterations for the wild type and G1439V proteins, and the predicted ddG was taken as the 412 difference between the wildtype and the mutant for the average of the three simulations.

\section{Commands are available upon request.}

414 Cell culture. 17Cl-1, Delayed brain tumor (DBT), 17Cl-1, L929, HeLa cells expressing the MHV 415 receptor carcinoembryonic antigen-related cell adhesion molecule 1 (CEACAM1) (HeLa-MHVR), and 416 baby hamster kidney cells expressing CEACAM1 (BHK-MVR) (all cell lines gifts provided by Stanley 417 Perlman, University of Iowa) were grown in Dulbecco's Modified Eagle Medium (DMEM) supplemented 
418 with $10 \%$ fetal bovine serum (FBS), $100 \mathrm{U} / \mathrm{ml}$ penicillin and $100 \mathrm{mg} / \mathrm{ml}$ streptomycin, HEPES, sodium

419 pyruvate, non-essential amino acids, and L-glutamine. Bone marrow-derived macrophages (BMDMs)

420 sourced from WT mice were differentiated by incubating cells in Roswell Park Memorial Institute

421 (RPMI) media supplemented with 10\% L929 cell supernatants, 10\% FBS, sodium pyruvate, $100 \mathrm{U} / \mathrm{ml}$

422 penicillin and $100 \mathrm{mg} / \mathrm{ml}$ streptomycin, and L-glutamine in for seven days. Cells were washed and

423 replaced with fresh media every day after the $4^{\text {th }}$ day.

424 Cell viability assay. BMDMs and 17Cl-1 cells were treated with the indicated compounds for 24 hours.

425 Cellular metabolic activity was assessed using a Vybrant MTT Cell Proliferation Assay (Thermo Fisher

426 Scientific) following manufacturer's instructions.

427 Mice. Pathogen-free C57BL/6 (B6) mice were originally purchased from Jackson Laboratories. Mice

428 were bred and maintained in the animal care facility at the University of Kansas as approved by the

429 University of Kansas Institutional Animal Care and Use Committee (IACUC) following guidelines set

430 forth in the Guide for the Care and Use of Laboratory Animals.

431 Generation of recombinant pBAC-JHMV constructs. All recombinant pBAC-JHMV constructs

432 were created using Red recombination (primers listed in Table 2). Recombinant WT ( $\mathrm{rJ}^{\mathrm{IA}}$ -

433 GFPrevN1347) and N1347A (rJ'IA-GFP-N1347A) MHV were previously described.

434 Recombinant BACs with the D1329A, G1439V, A1438T, and N1465A point mutations in the

435 nsp3 macrodomain were engineered using the Kan ${ }^{\mathrm{r}}$-I-SceI marker cassette for dual positive and

436 negative selection as previously described, using the primers listed in Table 2 (32). BAC DNA

437 from $\mathrm{Cml}^{\mathrm{r}} \mathrm{Kan}^{\mathrm{s}}$ colonies was analyzed by restriction enzyme digest, PCR, and direct sequencing

438 for isolation of correct clones. PCR and sequencing were done using the following primers

439 located just outside of the Mac1 gene sequence: F 5'-ggctgttgtggatggcaagca-3' and R 5'-

440 getttggtaccagcaacggag-3'. Wild-type repaired BACs were engineered by reintroducing the wild-

441 type sequence into the BAC clones containing the D1329A mutation using the same procedure as 
442 described above. The resulting BAC clones were termed pBAC-JHMVIA_GFP-D1329A, pBACJHMVIA_GFP-N1465A, pBAC-JHMVIA_GFP-D1329A/N1347A, pBAC-JHMVIA_GFP-G1439V, pBAC-JHMVIA_GFP-G1439V/A1438T, and pBAC-JHMVIA_GFP-repD1329.

Reconstitution of recombinant pBAC-JHMV-derived virus. Approximately $1 \times 10^{6} \mathrm{BHK}$ MVR cells were transfected with approximately $0.5-1 \mu \mathrm{g}$ of pBAC-JHMV DNA and $1 \mu \mathrm{g}$ of

447 pcDNA-MHV-N plasmid using Polyjet (SignaGen) as a transfection reagent. New recombinant

448 viruses used in this study were termed D1329A (rJ $\left.\mathrm{J}^{\mathrm{IA}}-\mathrm{GFP}-\mathrm{D} 1329 \mathrm{~A}\right), \mathrm{N} 1465 \mathrm{~A}\left(\mathrm{rJ}^{\mathrm{IA}}-\mathrm{GFP}-\right.$ N1465), G1439V (rJ ${ }^{\mathrm{IA}}-$ GFP-G1439V), A1438T/G1439V (rJ'IA-GFP- A1438T/G1439V), and

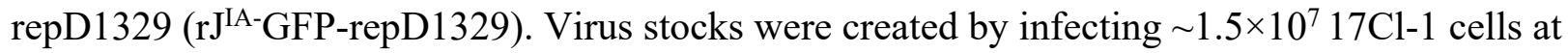
an MOI of 0.1 plaque-forming units (PFU)/cell and collecting both the cells and supernatant at

452 16-20 hpi. The cells were freeze-thawed, and debris was removed prior to collecting virus stocks.

453 Virus stocks were quantified by plaque assay on Hela-MHVR cells and sequenced by collecting

454 infected 17Cl-1 or L929 cells using TRIzol. RNA was isolated and cDNA was prepared using 455 MMLV-reverse transcriptase as per manufacturer's instructions (Thermo Fisher Scientific). The

456 Mac1 gene sequence was amplified by PCR using the same primers as described above for 457 sequencing BACs, and then resulting PCR products were sequenced by Sanger sequencing using 458 the forward primer. Sequence was analyzed using DNA Star software.

459 Virus infection. Cells were infected with recombinant virus at a multiplicity of infection (MOI) 460 of $0.05-0.1 \mathrm{PFU} /$ cell with a 45-60 min adsorption phase, unless otherwise stated. Olaparib 461 (APExBIO - Cat. \#A4154) and XAV-939 (APExBIO - Cat. \#A1877) were added to cells 462 following the adsorption phase at $10 \mu \mathrm{M}$. Nicotinomide Riboside Chloride (NR) (Chromadex) 463 was added to cells at $100 \mu \mathrm{M} 4 \mathrm{hr}$ prior to infection and then re-added immediately following the 464 adsorption phase of the infection. Male and female mice, 5 to 8 weeks old (unless otherwise 
465 indicated), were anesthetized with ketamine/xylazine and inoculated intranasally with either

$4663 \times 10^{3}$ or $3 \times 10^{4} \mathrm{PFU}$ of recombinant virus in a total volume of $12 \mu \mathrm{LMEM}$. To obtain viral

467 RNA from infected animals to sequence the virus following infection, mice were sacrificed, and

468 brain tissue was collected and homogenized in TRIzol (Invitrogen). RNA was isolated and

469 cDNA was prepared using MMLV-reverse transcriptase as per manufacturer's instructions

470 (Thermo Fisher Scientific). The macrodomain gene sequence was amplified by PCR and

471 sequenced as described above. Sequence was analyzed using DNA Star software.

472 Real-time qPCR analysis. RNA was isolated from BMDMs using TRIzol (Invitrogen) and

473 cDNA was prepared as described above. Quantitative real-time PCR (qRT-PCR) was performed

474 on a QuantStudio3 real-time PCR system using PowerUp SYBR Green Master Mix (Thermo

475 Fisher Scientific). Primers used for qPCR are listed in Table 3. Cycle threshold $\left(\mathrm{C}_{\mathrm{T}}\right)$ values were

476 normalized to the housekeeping gene hypoxanthine phosphoribosyltransferase (HPRT) by the

477 following equation: $\mathrm{C}_{\mathrm{T}}=\mathrm{C}_{\mathrm{T}(\text { gene of interest) }}-\mathrm{C}_{\mathrm{T}(\mathrm{HPRT})}$. Results are shown as a ratio to HPRT

478 calculated as $2^{-\Delta \mathrm{CT}}$.

479 Immunoblotting. Total cell extracts were lysed in sample buffer containing SDS, protease and

480 phosphatase inhibitors (Roche), $\beta$-mercaptoethanol, and a universal nuclease (Fisher Scientific).

481 Proteins were resolved on an SDS polyacrylamide gel, transferred to a polyvinylidene difluoride

482 (PVDF) membrane, hybridized with a primary antibody, reacted with an infrared (IR) dye-

483 conjugated secondary anti- body, visualized using a Li-COR Odyssey Imager (Li-COR), and

484 analyzed using Image Studio software. Primary antibodies used for immunoblotting included

485 anti-MHV N monoclonal antibody (52) and anti-actin monoclonal antibody (clone AC15;

486 Abcam, Inc.). Secondary IR antibodies were purchased from Li-COR. 
487 Statistical Analysis. All statistical analyses were done using an unpaired two-tailed student's t-

488 test to assess differences in mean values between groups, and graphs are expressed as geometric 489 mean \pm geometric SD (virus titers) or \pm SEM (qPCR). The $n$ value represents the number of

490 biologic replicates for each figure. All data was analyzed using GraphPad Prism software.

491 Significant $p$ values are denoted with $* \mathrm{p} \leq 0.05, * * \mathrm{p} \leq 0.01$, or $* * * \mathrm{p} \leq 0.001$.

\section{ACKNOWLEDGEMENTS}

This research was funded by the National Institutes of Health (NIH), grant numbers P20 GM113117, K22AI134993, and R35GM138029, and start-up funds from the University of

496 Kansas to A.R.F. C.M.K. was supported by the NIH Graduate Training at the Biology-Chemistry

497 Interface Grant T32GM132061.

499 Center), and Cohen (Oregon Health Sciences University) labs for insightful discussions on this

500 project; Stanley Perlman, Yousef Alhammad, and Srivatsan Parthisarathy for critical reading of

501 this manuscript; the Perlman lab for reagents; and Chromadex for supplying nicotinamide

502 riboside chloride (NR). 


\section{AUTHOR CONTRIBUTIONS}

505 Conceptualization: ARF, LSV

506 Data curation: LSV, JJOC, CMK, ARF, DKJ

507 Formal analysis: LSV, JJOC, ARF, DKJ

508 Funding acquisition: ARF

509 Investigation: LSV, JJOC, CMK, ED, NS, PS

510 Methodology: ARF

511 Project administration: ARF

512 Resources: ARF

513 Supervision: ARF

514 Validation: LSV, JJOC, CMK, ARF, DKJ

515 Visualization: LSV, JJOC, CMK, ARF, DKJ

516 Writing - original draft: LSV, ARF

517 Writing - review \& editing: all authors 
519 Table 1. MHV BAC Recovery Rates in BHK-MVR Cells

520

\begin{tabular}{lcc}
\hline BAC & Virus Recovered/Attempts & \% Recovery \\
\hline N1347A & $9 / 9$ & $100 \%$ \\
D1329A & $9 / 9$ & $100 \%$ \\
N1347A/D1329A & $0 / 12$ & $0 \%$ \\
G1439V & $4 / 11$ & $36.37 \%$ \\
A1438T/G1439V & $6 / 6$ & $100 \%$
\end{tabular}




\section{Table 2. Primers used to create recombinant BACs}

\begin{tabular}{|c|c|c|}
\hline Gene & Forward $5^{\prime} \rightarrow 3^{\prime}$ & Reverse $5^{\prime} \rightarrow 3^{\prime}$ \\
\hline D1329A & $\begin{array}{l}\text { ggttcatgtataacaccaaatgtttgttttgttaaagga } \\
\text { gatgttataaaggtttgcgcAGGATGACGA } \\
\text { CGATAAGTAGGG }\end{array}$ & $\begin{array}{l}\text { atgacttcagcaccaactctgcgcaaaacctttataacatc } \\
\text { tcctttaacaaaacaaacaGCCAGTGTTACAA } \\
\text { CCAATTAACC }\end{array}$ \\
\hline repD1329A & $\begin{array}{l}\text { ggttcatgtataacaccaaatgtttgttttgttaaagga } \\
\text { gctgttataaaggtttgcgcAGGATGACGA } \\
\text { CGATAAGTAGGG }\end{array}$ & $\begin{array}{l}\text { atgacttcagcaccaactctgcgcaaaacctttataacagc } \\
\text { tcctttaacaaaacaaacaGCCAGTGTTACAA } \\
\text { CCAATTAACC }\end{array}$ \\
\hline $\mathrm{N} 1465 \mathrm{~A}$ & $\begin{array}{l}\text { tctacttggtgtagtgacgaagaatgtcattcttgtcag } \\
\text { taataaccaggatgatttgaAGGATGACGA } \\
\text { CGATAAGTAGG }\end{array}$ & $\begin{array}{l}\text { cctgacacttctctatcacatcaaaatcatcctggttattact } \\
\text { gacaagaatgacattctGCCAGTGTTACAAC } \\
\text { CAATTAACC }\end{array}$ \\
\hline G1439V & $\begin{array}{l}\text { aataagtgtgacaatgttgtcaccactttaatttcggct } \\
\text { gttatatttagtgtgcctactAGGATGACGA } \\
\text { CGATAAGTAGGG }\end{array}$ & $\begin{array}{l}\text { agataagttaaggaaacatcagtaggcacactaaatataa } \\
\text { cagccgaaattaaagtggtgGCCAGTGTTACA } \\
\text { ACCAATTAACC }\end{array}$ \\
\hline A1438T/ & tattaataagtgtgacaatgttgtcaccactttaatttcg & aagttaaggaaacatcagtaggcacactaaattaacagtc \\
\hline G1439V & $\begin{array}{l}\text { actgttatatttagtgtgccAGGATGACGAC } \\
\text { GATAAGTAGGG }\end{array}$ & $\begin{array}{l}\text { gaaattaaagtggtgacaaGCCAGTGTTACAA } \\
\text { CCAATTAACC }\end{array}$ \\
\hline
\end{tabular}


TABLE 3. Quantitative real-time PCR primers

\begin{tabular}{lll}
\hline Gene & Forward $\mathbf{5}^{\prime} \rightarrow \mathbf{3}^{\prime}$ & Reverse $\mathbf{5} \boldsymbol{\prime} \rightarrow \mathbf{3}^{\prime}$ \\
\hline gRNA & AGGGAGTTTGACCTTGTTCAG & ATAATGCACCTGTCATCCTCG \\
IFN $\beta$ & TCAGAATGAGTGGTGGTTGC & GACCTTTCAAATGCAGTAGATTCA \\
HPRT & GCGTCGTGATTAGCGATGATG & CTCGAGCAAGTCTTTCAGTCC
\end{tabular}

523

524 


\section{REFERENCES}

1. Wang Y, Grunewald M, Perlman S. 2020. Coronaviruses: An Updated Overview of Their Replication and Pathogenesis. Methods Mol Biol 2203:1-29.

2. Lei J, Kusov Y, Hilgenfeld R. 2018. Nsp3 of coronaviruses: Structures and functions of a large multi-domain protein. Antiviral Res 149:58-74.

3. Allen MD, Buckle AM, Cordell SC, Lowe J, Bycroft M. 2003. The crystal structure of AF1521 a protein from Archaeoglobus fulgidus with homology to the non-histone domain of macroH2A. J Mol Biol 330:503-11.

4. Fehr AR, Jankevicius G, Ahel I, Perlman S. 2018. Viral Macrodomains: Unique Mediators of Viral Replication and Pathogenesis. Trends Microbiol 26:598-610.

5. Kusov Y, Tan J, Alvarez E, Enjuanes L, Hilgenfeld R. 2015. A G-quadruplex-binding macrodomain within the "SARS-unique domain" is essential for the activity of the SARS-coronavirus replication-transcription complex. Virology 484:313-322.

6. Srinivasan S, Cui H, Gao Z, Liu M, Lu S, Mkandawire W, Narykov O, Sun M, Korkin D. 2020. Structural Genomics of SARS-CoV-2 Indicates Evolutionary Conserved Functional Regions of Viral Proteins. Viruses 12(4):360.

7. Chatterjee A, Johnson MA, Serrano P, Pedrini B, Joseph JS, Neuman BW, Saikatendu K, Buchmeier MJ, Kuhn P, Wuthrich K. 2009. Nuclear magnetic resonance structure shows that the severe acute respiratory syndrome coronavirus-unique domain contains a macrodomain fold. J Virol 83:1823-36.

8. Johnson MA, Chatterjee A, Neuman BW, Wuthrich K. 2010. SARS coronavirus unique domain: three-domain molecular architecture in solution and RNA binding. J Mol Biol 400:724-42.

9. Ma-Lauer Y, Carbajo-Lozoya J, Hein MY, Muller MA, Deng W, Lei J, Meyer B, Kusov Y, von Brunn B, Bairad DR, Hunten S, Drosten C, Hermeking H, Leonhardt H, Mann M, Hilgenfeld R, von Brunn A. 2016. p53 down-regulates SARS coronavirus replication and is targeted by the SARS-unique domain and PLpro via E3 ubiquitin ligase RCHY1. Proc Natl Acad Sci U S A 113:E5192-201.

10. Tan J, Kusov Y, Mutschall D, Tech S, Nagarajan K, Hilgenfeld R, Schmidt CL. 2007. The "SARS-unique domain" (SUD) of SARS coronavirus is an oligo(G)-binding protein. Biochem Biophys Res Commun 364:877-82.

11. Tan J, Vonrhein C, Smart OS, Bricogne G, Bollati M, Kusov Y, Hansen G, Mesters JR, Schmidt CL, Hilgenfeld R. 2009. The SARS-unique domain (SUD) of SARS coronavirus contains two macrodomains that bind G-quadruplexes. PLoS Pathog 5:e1000428.

12. Fehr AR, Singh SA, Kerr CM, Mukai S, Higashi H, Aikawa M. 2020. The impact of PARPs and ADP-ribosylation on inflammation and host-pathogen interactions. Genes Dev 34:341-359.

13. Li L, Zhao H, Liu P, Li C, Quanquin N, Ji X, Sun N, Du P, Qin CF, Lu N, Cheng G. 2018. PARP12 suppresses Zika virus infection through PARP-dependent degradation of NS1 and NS3 viral proteins. Sci Signal 11.

14. Rack JGM, Palazzo L, Ahel I. 2020. (ADP-ribosyl)hydrolases: structure, function, and biology. Genes Dev 34:263-284.

15. Egloff MP, Malet H, Putics A, Heinonen M, Dutartre H, Frangeul A, Gruez A, Campanacci V, Cambillau C, Ziebuhr J, Ahola T, Canard B. 2006. Structural and 
functional basis for ADP-ribose and poly(ADP-ribose) binding by viral macro domains. J Virol 80:8493-502.

16. Fehr AR, Channappanavar R, Jankevicius G, Fett C, Zhao J, Athmer J, Meyerholz DK, Ahel I, Perlman S. 2016. The Conserved Coronavirus Macrodomain Promotes Virulence and Suppresses the Innate Immune Response during Severe Acute Respiratory Syndrome Coronavirus Infection. MBio 7(6):e01721-16.

17. Jankevicius G, Hassler M, Golia B, Rybin V, Zacharias M, Timinszky G, Ladurner AG. 2013. A family of macrodomain proteins reverses cellular mono-ADP-ribosylation. Nat Struct Mol Biol 20:508-14.

18. Putics A, Filipowicz W, Hall J, Gorbalenya AE, Ziebuhr J. 2005. ADP-ribose-1"monophosphatase: a conserved coronavirus enzyme that is dispensable for viral replication in tissue culture. J Virol 79:12721-31.

19. Alhammad YMO, Fehr AR. 2020. The Viral Macrodomain Counters Host Antiviral ADP-Ribosylation. Viruses 12(4):384.

20. Cho CC, Lin MH, Chuang CY, Hsu CH. 2016. Macro Domain from Middle East Respiratory Syndrome Coronavirus (MERS-CoV) Is an Efficient ADP-ribose Binding Module: CRYSTAL STRUCTURE AND BIOCHEMICAL STUDIES. J Biol Chem 291:4894-902.

21. Eriksson KK, Cervantes-Barragan L, Ludewig B, Thiel V. 2008. Mouse hepatitis virus liver pathology is dependent on ADP-ribose-1"-phosphatase, a viral function conserved in the alpha-like supergroup. J Virol 82:12325-34.

22. Fehr AR, Athmer J, Channappanavar R, Phillips JM, Meyerholz DK, Perlman S. 2015. The nsp3 macrodomain promotes virulence in mice with coronavirus-induced encephalitis. J Virol 89:1523-36.

23. Grunewald ME, Chen Y, Kuny C, Maejima T, Lease R, Ferraris D, Aikawa M, Sullivan CS, Perlman S, Fehr AR. 2019. The coronavirus macrodomain is required to prevent PARP-mediated inhibition of virus replication and enhancement of IFN expression. PLoS Pathog 15(5):e1007756.

24. Abraham R, Hauer D, McPherson RL, Utt A, Kirby IT, Cohen MS, Merits A, Leung AKL, Griffin DE. 2018. ADP-ribosyl-binding and hydrolase activities of the alphavirus nsP3 macrodomain are critical for initiation of virus replication. Proc Natl Acad Sci U S A 115:E10457-E10466.

25. Abraham R, McPherson RL, Dasovich M, Badiee M, Leung AKL, Griffin DE. 2020. Both ADP-Ribosyl-Binding and Hydrolase Activities of the Alphavirus nsP3 Macrodomain Affect Neurovirulence in Mice. mBio 11(1):e03253-19.

26. Saikatendu KS, Joseph JS, Subramanian V, Clayton T, Griffith M, Moy K, Velasquez J, Neuman BW, Buchmeier MJ, Stevens RC, Kuhn P. 2005. Structural basis of severe acute respiratory syndrome coronavirus ADP-ribose-1"-phosphate dephosphorylation by a conserved domain of nsP3. Structure 13:1665-75.

27. Xu Y, Cong L, Chen C, Wei L, Zhao Q, Xu X, Ma Y, Bartlam M, Rao Z. 2009. Crystal structures of two coronavirus ADP-ribose-1"-monophosphatases and their complexes with ADP-Ribose: a systematic structural analysis of the viral ADRP domain. J Virol 83:1083-92.

28. Karras GI, Kustatscher G, Buhecha HR, Allen MD, Pugieux C, Sait F, Bycroft M, Ladurner AG. 2005. The macro domain is an ADP-ribose binding module. EMBO J 24:1911-20. 
29. McPherson RL, Abraham R, Sreekumar E, Ong SE, Cheng SJ, Baxter VK, Kistemaker HA, Filippov DV, Griffin DE, Leung AK. 2017. ADP-ribosylhydrolase activity of Chikungunya virus macrodomain is critical for virus replication and virulence. Proc Natl Acad Sci U S A 114:1666-1671.

30. Alhammad YMO, Kashipathy MM, Roy A, Gagne JP, McDonald P, Gao P, Nonfoux L, Battaile KP, Johnson DK, Holmstrom ED, Poirier GG, Lovell S, Fehr AR. 2020. The SARS-CoV-2 conserved macrodomain is a mono-ADP-ribosylhydrolase. J Virol 95(3):e01969-20.

31. Michalska K, Kim Y, Jedrzejczak R, Maltseva NI, Stols L, Endres M, Joachimiak A. 2020. Crystal structures of SARS-CoV-2 ADP-ribose phosphatase: from the apo form to ligand complexes. IUCrJ 7:814-824.

32. Fehr AR. 2020. Bacterial Artificial Chromosome-Based Lambda Red Recombination with the I-SceI Homing Endonuclease for Genetic Alteration of MERS-CoV. Methods Mol Biol 2099:53-68.

33. Eckei L, Krieg S, Butepage M, Lehmann A, Gross A, Lippok B, Grimm AR, Kummerer BM, Rossetti G, Luscher B, Verheugd P. 2017. The conserved macrodomains of the nonstructural proteins of Chikungunya virus and other pathogenic positive strand RNA viruses function as mono-ADP-ribosylhydrolases. Sci Rep 7:41746.

34. Deng X, Mettelman RC, O'Brien A, Thompson JA, O'Brien TE, Baker SC. 2019. Analysis of Coronavirus Temperature-Sensitive Mutants Reveals an Interplay between the Macrodomain and Papain-Like Protease Impacting Replication and Pathogenesis. J Virol 93(12):e02140-18.

35. Trammell SA, Schmidt MS, Weidemann BJ, Redpath P, Jaksch F, Dellinger RW, Li Z, Abel ED, Migaud ME, Brenner C. 2016. Nicotinamide riboside is uniquely and orally bioavailable in mice and humans. Nat Commun 7:12948.

36. Trammell SA, Weidemann BJ, Chadda A, Yorek MS, Holmes A, Coppey LJ, Obrosov A, Kardon RH, Yorek MA, Brenner C. 2016. Nicotinamide Riboside Opposes Type 2 Diabetes and Neuropathy in Mice. Sci Rep 6:26933.

37. Heer CD, Sanderson DJ, Voth LS, Alhammad YMO, Schmidt MS, Trammell SAJ, Perlman S, Cohen MS, Fehr AR, Brenner C. 2020. Coronavirus infection and PARP expression dysregulate the NAD metabolome: An actionable component of innate immunity. J Biol Chem 295:17986-17996.

38. Bergmann CC, Lane TE, Stohlman SA. 2006. Coronavirus infection of the central nervous system: host-virus stand-off. Nat Rev Microbiol 4:121-32.

39. Rack JGM, Zorzini V, Zhu Z, Schuller M, Ahel D, Ahel I. 2020. Viral macrodomains: a structural and evolutionary assessment of the pharmacological potential. Open Biol 10:200237.

40. Schuller M, Correy GJ, Gahbauer S, Fearon D, Wu T, Diaz RE, Young ID, Martins LC, Smith DH, Schulze-Gahmen U, Owens TW, Deshpande I, Merz GE, Thwin AC, Biel JT, Peters JK, Moritz M, Herrera N, Kratochvil HT, Aimon A, Bennett JM, Neto JB, Cohen AE, Dias A, Douangamath A, Dunnett L, Fedorov O, Ferla MP, Fuchs M, Gorrie-Stone TJ, Holton JM, Johnson MG, Krojer T, Meigs G, Powell AJ, Rack JGM, Rangel VL, Russi S, Skyner RE, Smith CA, Soares AS, Wierman JL, Zhu K, Jura N, Ashworth A, Irwin J, Thompson MC, Gestwicki JE, von Delft F, Shoichet BK, et al. 2020. Fragment Binding to the Nsp3 Macrodomain of SARS-CoV-2 Identified Through Crystallographic Screening and Computational Docking. bioRxiv doi:10.1101/2020.11.24.393405. 
661 41. Babar Z, Khan M, Zahra M, Anwar M, Noor K, Hashmi HF, Suleman M, Waseem M,

662

663

664

665

666

667

668

669

670

671

672

673

674

675

676

677

678

679

680

681

682

683

684

685

686

687

688

689

690

691

692

693

694

695

696

697

698

699

Shah A, Ali S, Ali SS. 2020. Drug similarity and structure-based screening of medicinal compounds to target macrodomain-I from SARS-CoV-2 to rescue the host immune system: a molecular dynamics study. J Biomol Struct Dyn doi:10.1080/07391102.2020.1815583:1-15.

42. Hussain I, Pervaiz N, Khan A, Saleem S, Shireen H, Wei DQ, Labrie V, Bao Y, Abbasi AA. 2020. Evolutionary and structural analysis of SARS-CoV-2 specific evasion of host immunity. Genes Immun doi:10.1038/s41435-020-00120-6.

43. Virdi RS, Bavisotto RV, Hopper NC, Vuksanovic N, Melkonian TR, Silvaggi NR, Frick DN. 2020. Discovery of Drug-Like Ligands for the Mac1 Domain of SARS-CoV-2 Nsp3. SLAS Discov 25:1162-1170.

44. Lin MH, Chang SC, Chiu YC, Jiang BC, Wu TH, Hsu CH. 2020. Structural, Biophysical, and Biochemical Elucidation of the SARS-CoV-2 Nonstructural Protein 3 Macro Domain. ACS Infect Dis 6:2970-2978.

45. Park E, Griffin DE. 2009. The nsP3 macro domain is important for Sindbis virus replication in neurons and neurovirulence in mice. Virology 388:305-14.

46. Kleine H, Poreba E, Lesniewicz K, Hassa PO, Hottiger MO, Litchfield DW, Shilton BH, Luscher B. 2008. Substrate-assisted catalysis by PARP10 limits its activity to monoADP-ribosylation. Mol Cell 32:57-69.

47. Buch-Larsen SC, Hendriks IA, Lodge JM, Rykaer M, Furtwangler B, Shishkova E, Westphall MS, Coon JJ, Nielsen ML. 2020. Mapping Physiological ADP-Ribosylation Using Activated Ion Electron Transfer Dissociation. Cell Rep 32:108176.

48. Grunewald ME, Fehr AR, Athmer J, Perlman S. 2018. The coronavirus nucleocapsid protein is ADP-ribosylated. Virology 517:62-68.

49. Song Y, DiMaio F, Wang RY, Kim D, Miles C, Brunette T, Thompson J, Baker D. 2013. High-resolution comparative modeling with RosettaCM. Structure 21:1735-42.

50. Leaver-Fay A, Tyka M, Lewis SM, Lange OF, Thompson J, Jacak R, Kaufman K, Renfrew PD, Smith CA, Sheffler W, Davis IW, Cooper S, Treuille A, Mandell DJ, Richter F, Ban YE, Fleishman SJ, Corn JE, Kim DE, Lyskov S, Berrondo M, Mentzer S, Popovic Z, Havranek JJ, Karanicolas J, Das R, Meiler J, Kortemme T, Gray JJ, Kuhlman B, Baker D, Bradley P. 2011. ROSETTA3: an object-oriented software suite for the simulation and design of macromolecules. Methods Enzymol 487:545-74.

51. Park H, Bradley P, Greisen P, Jr., Liu Y, Mulligan VK, Kim DE, Baker D, DiMaio F. 2016. Simultaneous Optimization of Biomolecular Energy Functions on Features from Small Molecules and Macromolecules. J Chem Theory Comput 12:6201-6212.

52. Perlman S, Schelper R, Ries D. 1987. Maternal antibody-modulated MHV-JHM infection in C57BL/6 and BALB/c mice. Adv Exp Med Biol 218:297-305. 


\section{FIGURE LEGENDS}

701 Figure 1. Rosetta-derived structure of the MHV Mac1 protein. (A) Cartoon representation of

702 MHV Mac-1 with ADP-ribose as determined by Rosetta. (B) Hydrogen bond interactions

703 (dashed lines) between ADP-ribose and amino acids with modeled water molecules. (C-D)

704 Superposition of MHV Mac1 (green) (6WOJ) with other CoV Mac1 structures. (C) SARS-CoV-

7052 Mac1 with ADP-ribose (magenta) (6WOJ) and (D) MERS-CoV Mac1 with ADP-ribose (cyan)

706 (5DUS). (E-F) Superposition of MHV Mac1 (green) with other CoV Mac1 structures

707 highlighting the ADP-ribose binding site. (E) SARS-CoV-2 (magenta), (F) MERS-CoV (cyan).

708 The ADP-ribose molecules are colored gray for MHV (A-F) and are rendered as orange

709 cylinders for SARS-CoV-2 Mac1 (C,E) and blue cylinders for MERS-CoV Mac1 (D,F).

710 Conserved waters are shown as red spheres.

711 Figure 2. D1329A, but not N1465A, is highly attenuated in both primary cells and in cell lines.

712 BMDM (A), 17Cl-1 (B-D), L929 (E-F), and DBT (G-H) cells were infected with WT, N1347A,

713 D1329A, N1465A, and repD1329 viruses as described in Materials \& Methods. Progeny virus

714 was collected at indicated time points and virus titers were determined by plaque assay. In

715 addition, cell lysates were collected and viral protein levels were determined by immunoblotting.

716 The data show one experiment representative of at least two independent experiments (A-C,E,G)

717 with $n=4(A)$ or $n=3(B, C, E, G)$ in each experiment. The data in $(D, F, H)$ show one experiment

718 representative of two independent experiments.

719 Figure 3. D1329A infection results in lower IFN- $\beta$ mRNA levels than N1347A infection in

720 BMDMs. (A-B) BMDMs were infected with WT, N1347A, D1329A, or N1465A recombinant

721 virus. Cells were collected at the indicated times post infection and RNA was purified. gRNA

722 (A) and IFN- $\beta$ (B) mRNA levels were determined by RT-qPCR using primers listed in Table 2 
723 and normalized to HPRT mRNA levels. The data in (A and B) show one experiment

724 representative of four independent experiments with $\mathrm{n}=4$ for each experiment.

725 Figure 4. D1329A replication is significantly increased by the addition of PARP inhibitors. (A-

726 B) BMDMs (A) and 17Cl-1 cells (B) were treated with indicated compounds, and at 24 hours,

727 cell viability was measured using an MTT assay as described in Materials \& Methods. (C-D) WT

728 BMDMs (C) and 17Cl-1 cells (D) were infected with WT, N1347A, or D1329A, and then treated

729 with $0.25 \%$ DMSO, $10 \mu \mathrm{M}$ Olaparib (2281), or $10 \mu \mathrm{M}$ XAV-939 as described in Materials \&

730 Methods. Progeny virus was collected at indicated time points and virus titers were determined

731 by plaque assay. The data in (A-D) show one experiment representative of two independent

732 experiments with $n=4(A, B)$ or $n=3(C, D)$ for each experiment.

733 Figure 5. The addition of NR, a precursor of the PARP substrate NAD ${ }^{+}$, further decreases the

734 replication of D1329A. (A-B) BMDMs (A) and 17Cl-1 cells (B) were treated with NR as

735 described above, and at 24 hours cell viability was measured using an MTT assay as described in

736 Methods. (C-D) WT BMDMs (C) and 17Cl-1 cells (D) were either mock treated $\left(\mathrm{H}_{2} \mathrm{O}\right)$ or treated

737 with NR as described in Materials \& Methods. Progeny virus was collected at indicated time

738 points and virus titers were determined by plaque assay. The data in (A-D) show one experiment

739 representative of two independent experiments with $n=4(A, B)$ or $n=3(C, D)$ for each

740 experiment.

741 Figure 6. D1329A, but not N1465A, is highly attenuated in vivo. (A-B) WT male and female B6

742 mice were infected with $3 \times 10^{4}$ PFU of WT, N1347A, and N1465A intranasally and monitored

743 for survival and weight loss daily for 12 days. The data show the combined results of two

744 independent experiments. WT, $\mathrm{n}=5$ for male and female mice; N1347A, $\mathrm{n}=5$ for male and female

745 mice; N1465A, n=4 for male and female mice. (C-D) WT male and female B6 mice were 
infected with $3 \times 10^{3}$ PFU of WT and D1329A as described above. The data show the combined

747 results of two independent experiments. WT, $\mathrm{n}=5$ for male and female mice; D1329A, $\mathrm{n}=7$ for

male and $\mathrm{n}=6$ for female mice. (E) D1329A virus readily reverts in vivo. The brains of 3 mice (2

male (M); 1 female (F)) which succumbed to infection with D1329A were harvested and their

viral RNA was amplified by RT-PCR with Mac1 specific primers. The PCR product was

sequenced by Sanger sequencing and analyzed using DNA Star software. Mouse \#1 and \#3

reverted to wild-type virus sequence, while mouse \#2 evolved 2 new mutations, L1313V \&

753 K1327E.

754 Figure 7. The A1438T/G1439V virus, a revertant of G1439V, is recoverable but replicates at

755 slightly reduced levels compared to WT virus and is attenuated in vivo. (A-B) L929 cells were

756 infected with WT, D1329A, and AG1438/1439TV viruses as described in Materials \& Methods.

757 Progeny virus was collected at $24 \mathrm{hpi}$ and virus titers were determined by plaque assay. In

758 addition, cell lysates were collected at 24 hpi and viral protein levels were determined by

759 immunoblotting. The data in (A) shows one experiment representative of two independent

760 experiments with $n=3$ for each experiment. The data in (B) shows one experiment representative

761 of two independent experiments. (C-D) WT male B6 mice were infected with $3 \times 10^{3}$ PFU of WT

762 and A1438T/G1439V intranasally and monitored for survival (C) and weight loss (D) daily for

76312 days. WT, $n=3$; A1438T/G1439V, n=6. (D-E) Rosetta predicted structures of WT MERS-

764 CoV Mac1 around the distal ribose compared to G128V (E) and A127T/G128V (F) proteins.

765 G128V (orange cylinders) is predicted to cause a disruption of water molecules (E) (WT - red

766 spheres; G128V or A127T/G128V - black spheres). The A127T mutation (salmon cylinders) is

767 predicted to restore this water network back to their original location (F), likely restoring critical

768 hydrogen bonds. 
A

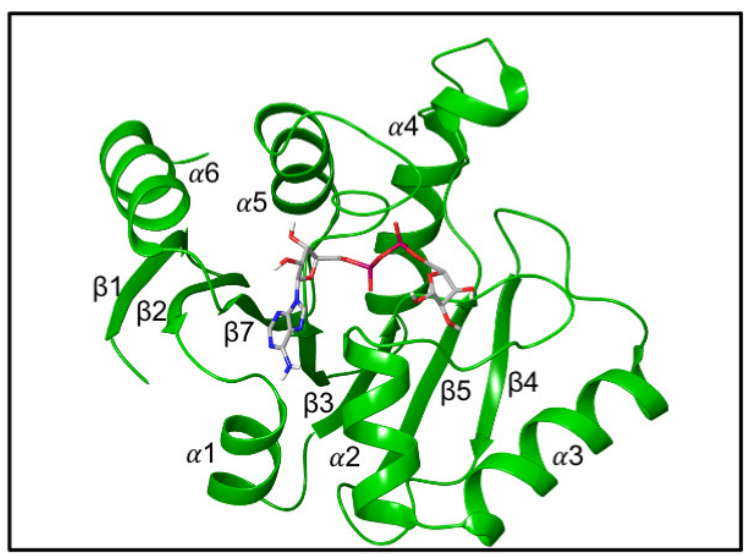

C

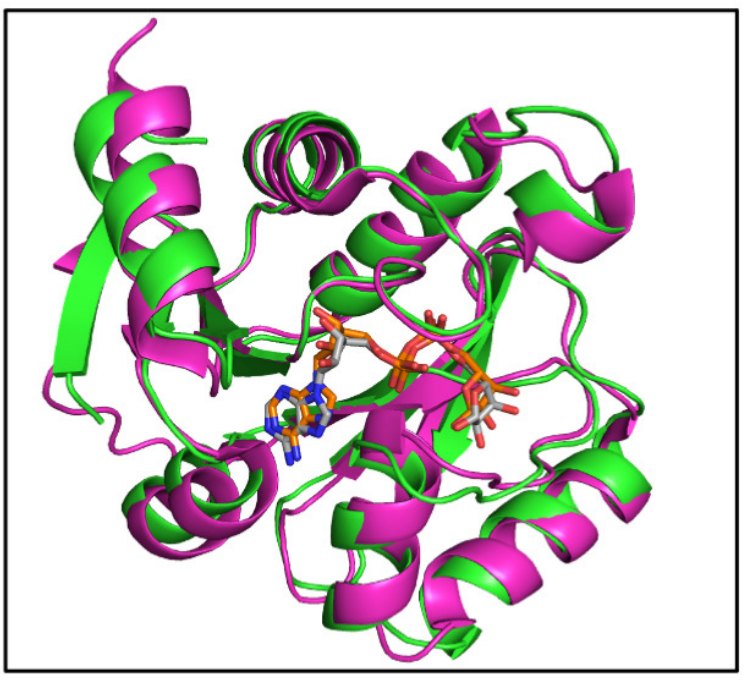

E

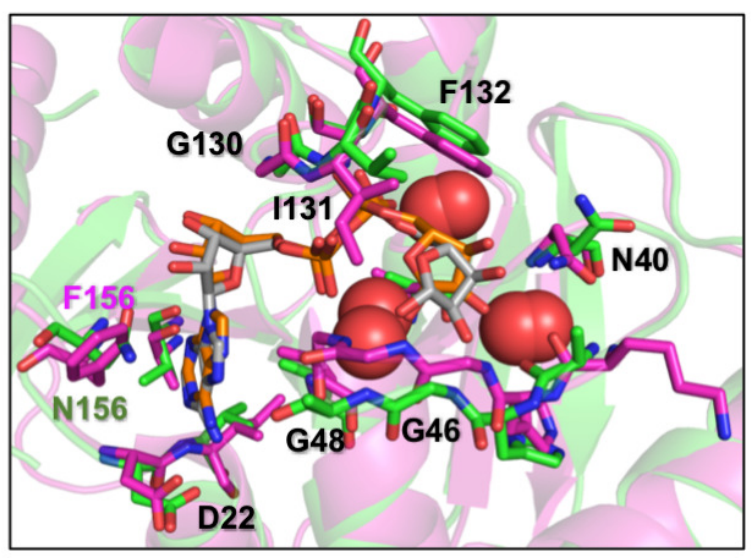

B

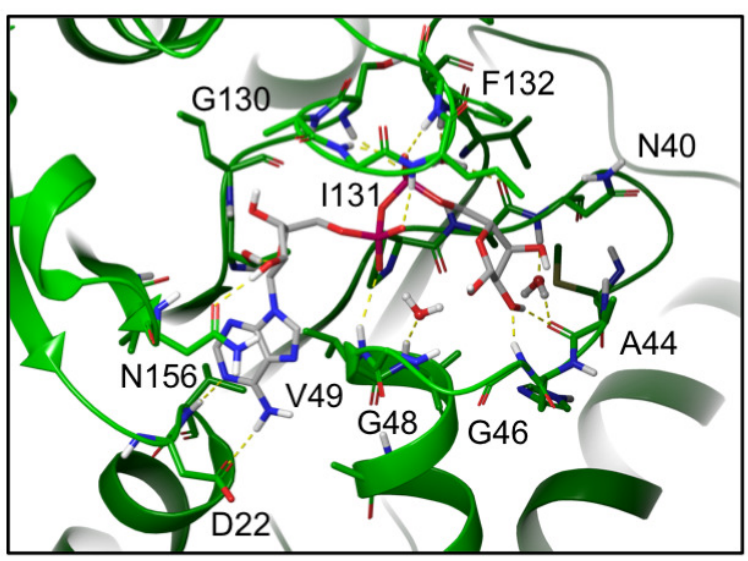

D

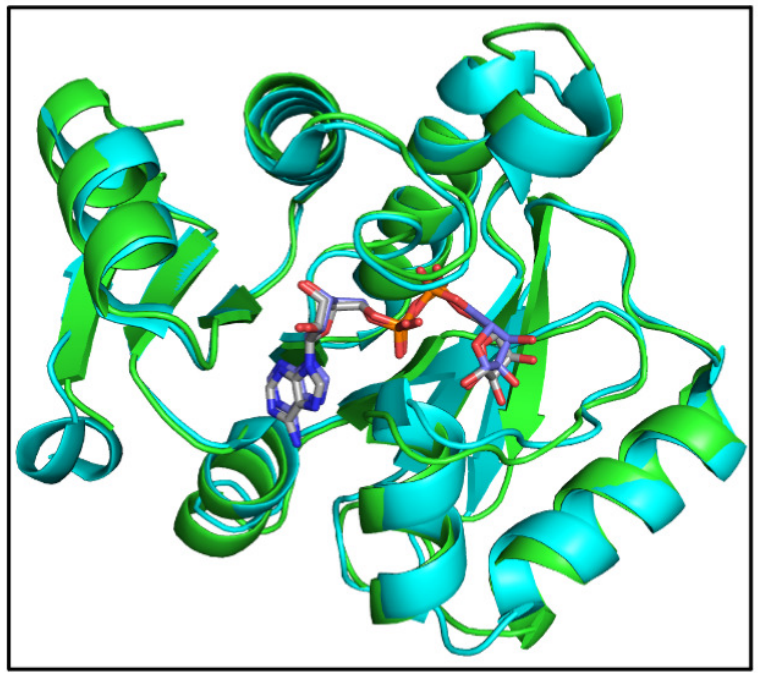

$\mathbf{F}$

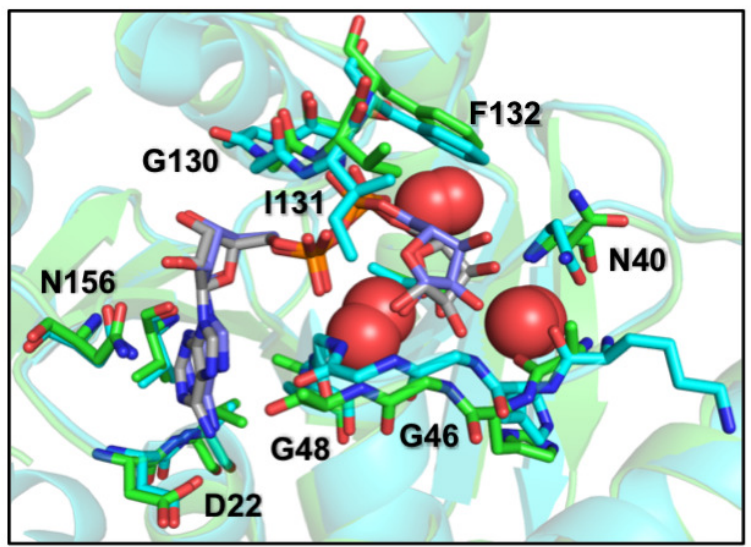

Figure 1. Rosetta-derived structure of the MHV Mac1 protein. A) Cartoon representation of MHV Mac1 with ADP-ribose. B) Hydrogen bond interactions (dashed lines) between ADP-ribose and amino acids with modeled water molecules. C-D) Superposition of MHV macrodomain (green) (6WOJ) with coronavirus macrodomain structures. C) SARS-CoV-2 Mac1 with ADP-ribose (magenta) (6WOJ) and D) MERS-CoV Mac1 with ADP-ribose (cyan) (5DUS). E-F) Superposition of MHV Mac1 (green) with other coronavirus Mac1 structures highlighting the ADP-ribose binding site. E) SARS-CoV-2 (magenta), F) MERS-CoV (cyan). The ADP-ribose molecules are colored gray for MHV (A-F) and are rendered as orange cylinders for SARS-CoV-2 Mac1 (C,E) and blue cylinders for MERS-CoV Mac1 (D,F). Conserved waters are shown as red spheres. 


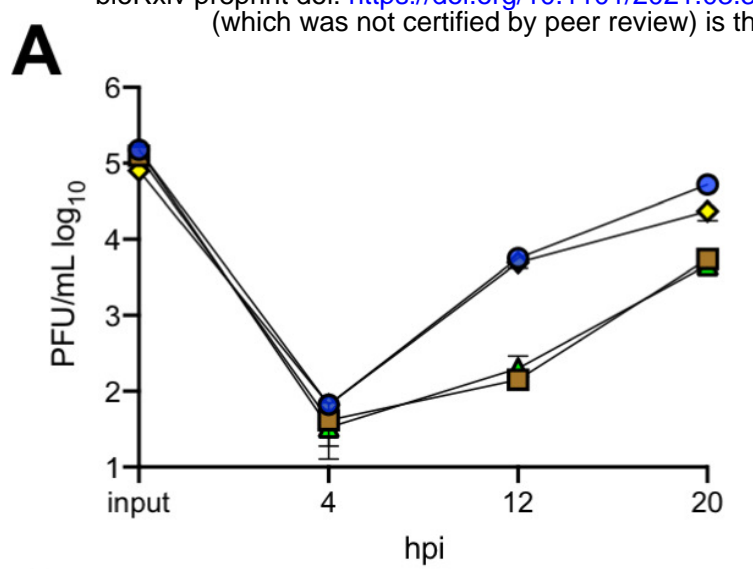

C

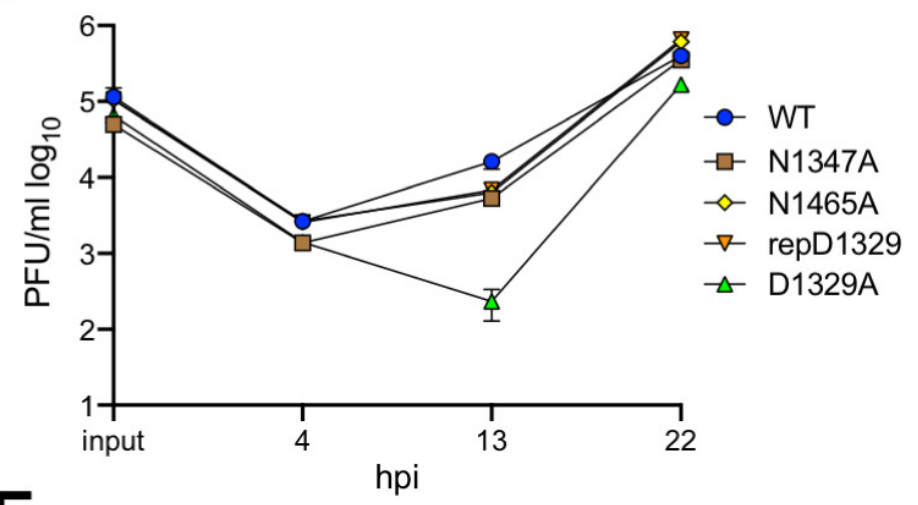

$\mathbf{E}$

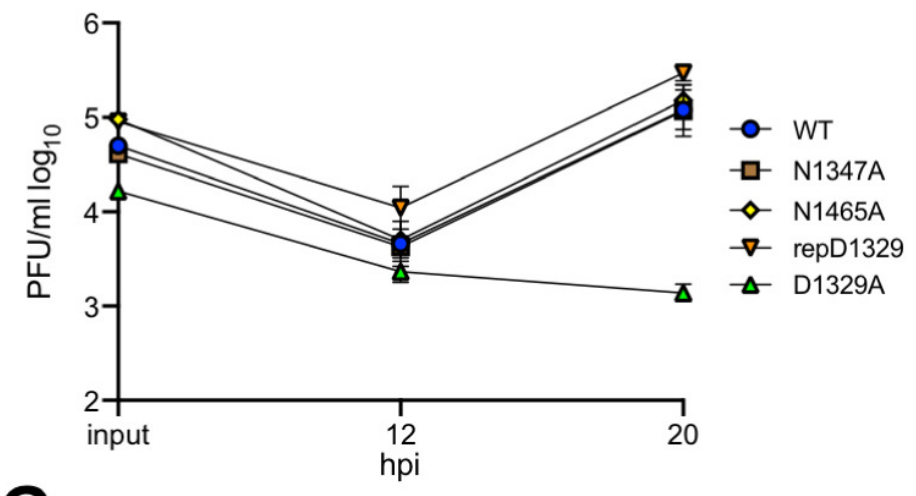

$\mathbf{G}$

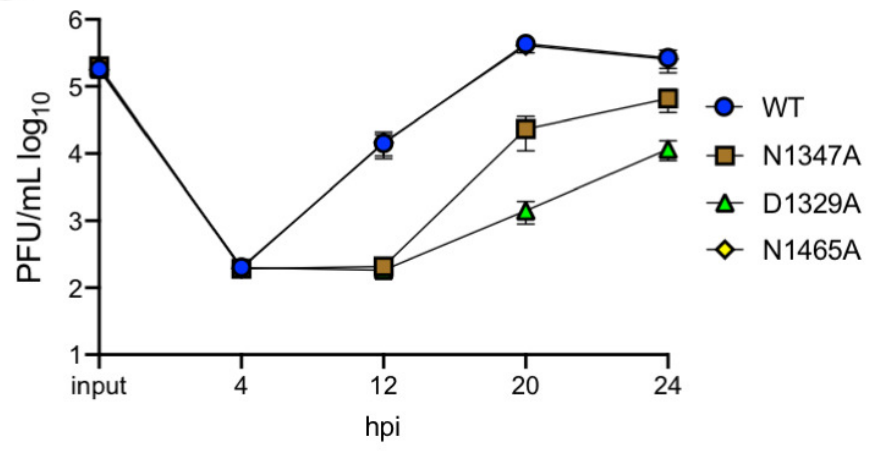

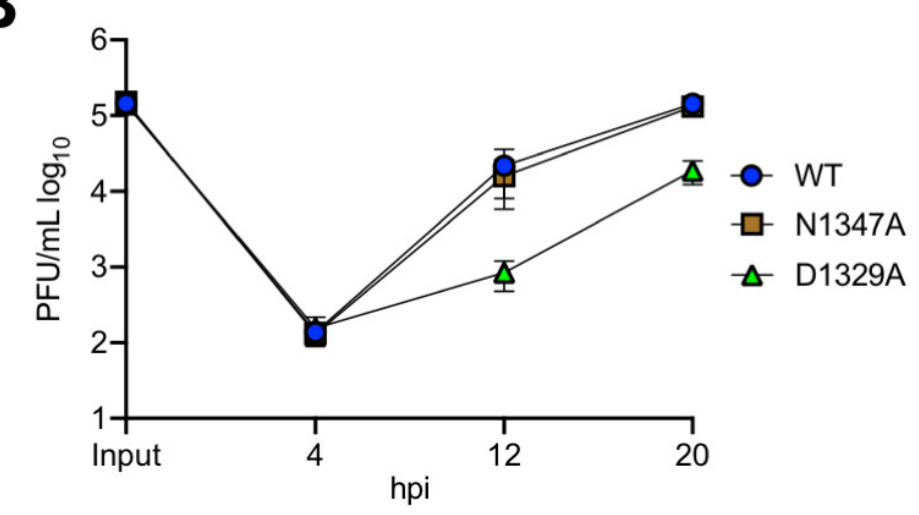

D

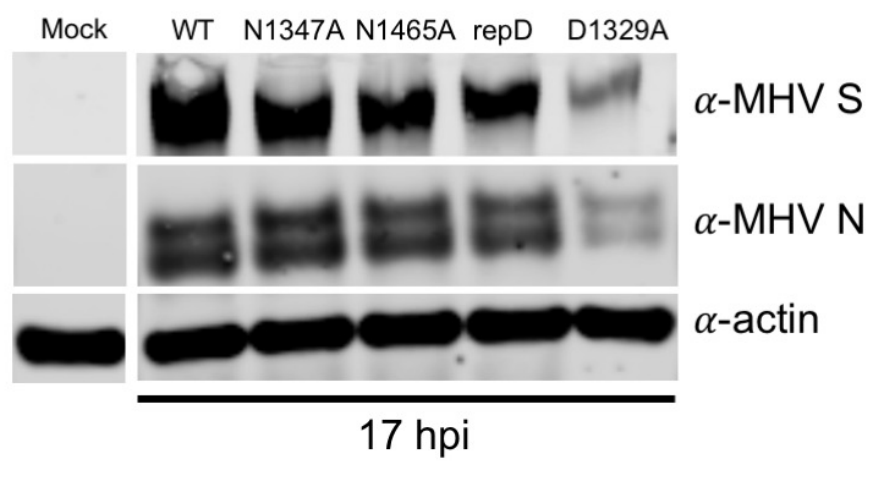

$\mathbf{F}$

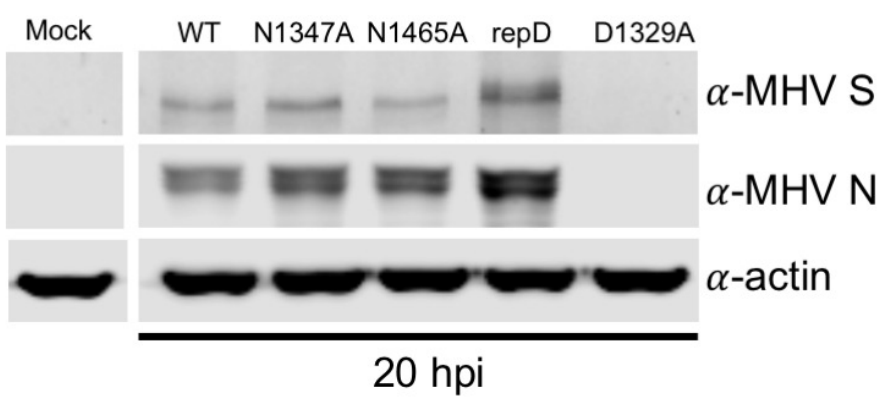

H

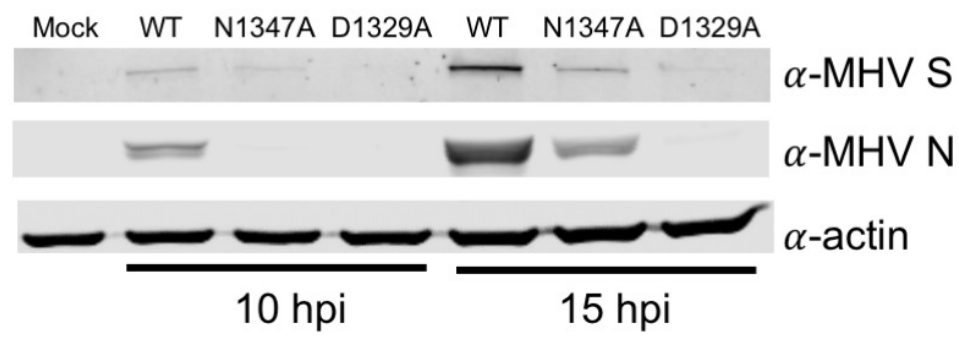

Figure 2. D1329A, but not N1465A, is highly attenuated in both primary cells and in transformed cell lines. BMDM (A), 17CL-1 (B-D), L929 (E-F), and DBT (G-H) cells were infected with WT, N1347A, D1329A, N1465A, and repD1329 viruses as described in Materials \& Methods. Progeny virus was collected at indicated time points and virus titers were determined by plaque assay (A,B,C,E,G), and viral protein levels were determined by immunoblotting $(\mathrm{D}, \mathrm{F}, \mathrm{H})$. The data show one experiment representative of at least two independent experiments $(A-C, E, G)$ with $n=4(A)$ or $n=3(B, C, E, G)$ in each experiment. The data in $(D, F, H)$ show one experiment representative of two independent experiments. 

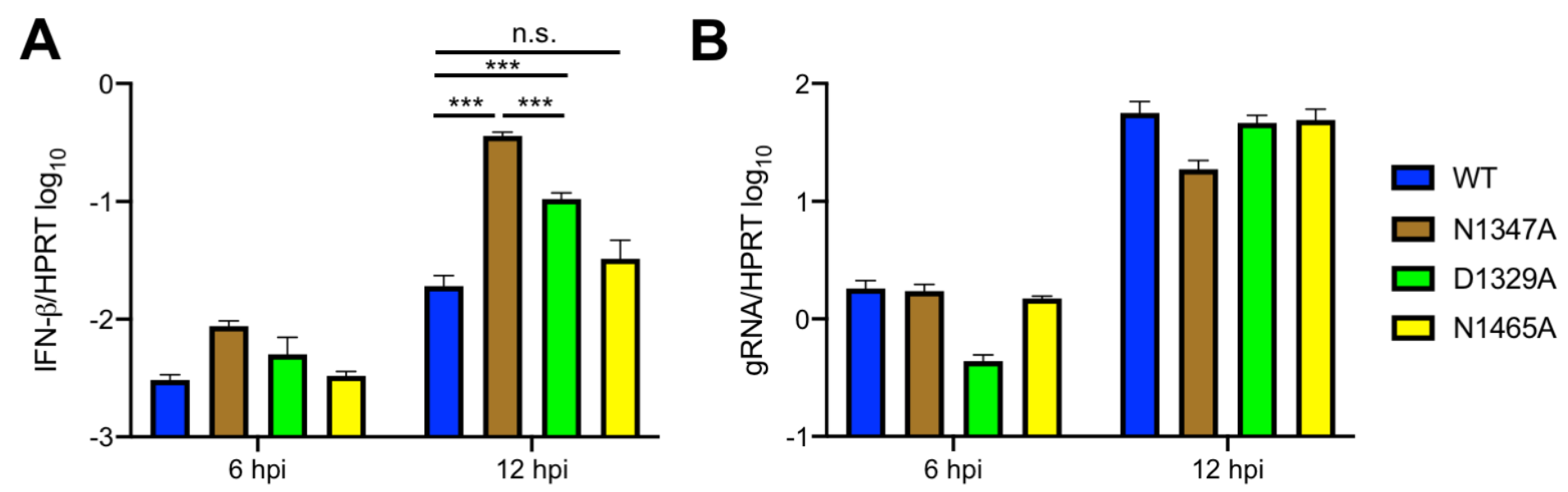

Figure 3. D1329A infection results in lower IFN- $\beta$ mRNA levels than N1347A infection in BMDMs. (A-B) BMDMs were infected with WT, N1347A, D1329A, or N1465A recombinant virus. Cells were collected at the indicated times post infection and RNA was purified. gRNA (A) and IFN- $\beta$ (B) mRNA levels were determined by RT-qPCR using primers listed in Table 2 and normalized to HPRT mRNA levels. The data in (A and B) show one experiment representative of four independent experiments with $n=4$ for each experiment. 

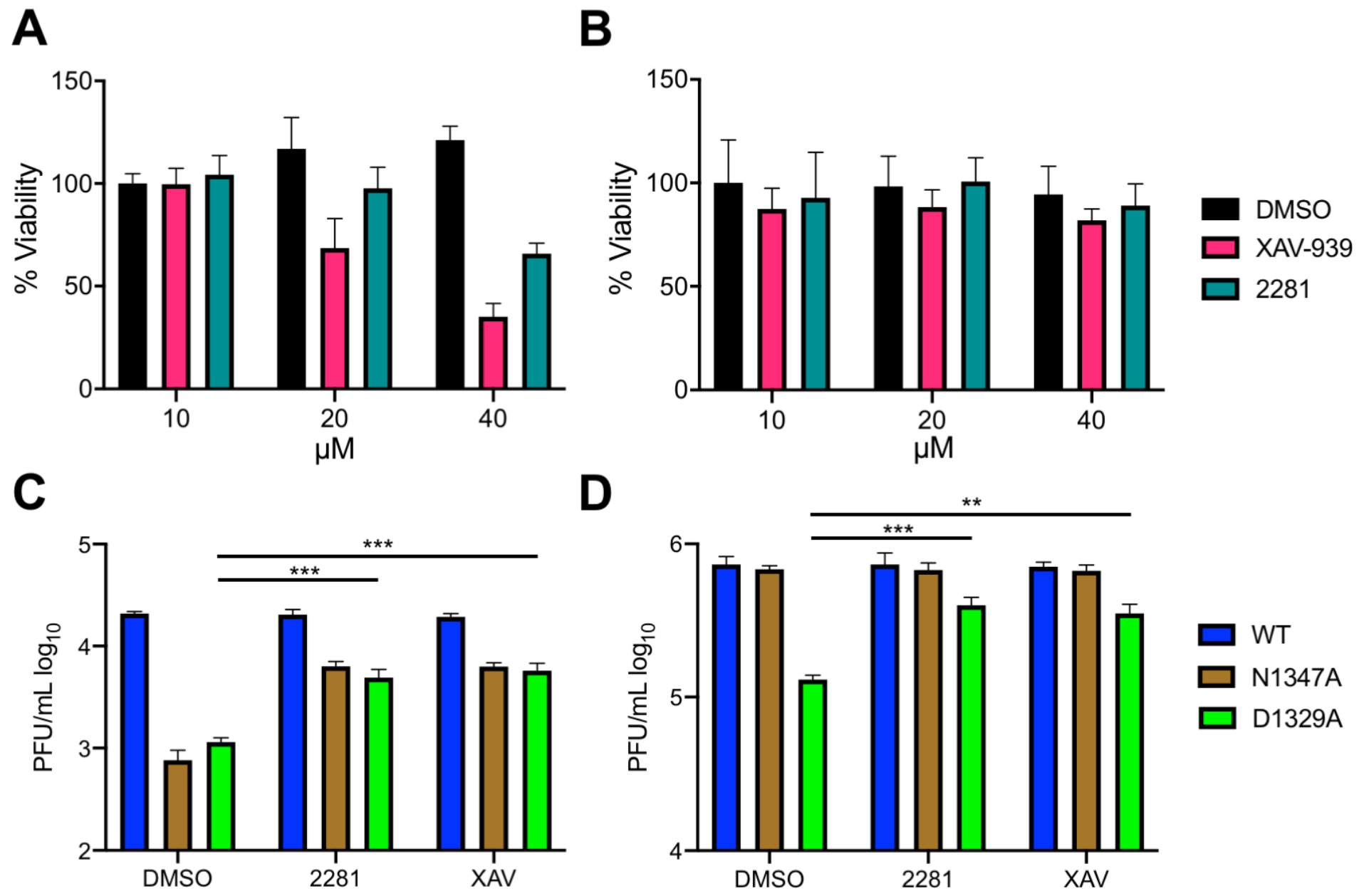

Figure 4. D1329A replication is significantly increased by the addition of PARP inhibitors. (A-B) BMDMs (A) and 17Cl-1 cells (B) were treated with indicated compounds, and at 24 hours, cell viability was measured using an MTT assay as described in Materials \& Methods. (C-D) WT BMDMs (C) and 17Cl-1 cells (D) were infected with WT, N1347A, or D1329A, and then treated with 0.25\% DMSO, $10 \mu$ M Olaparib (2281), or 10 $\mu \mathrm{M}$ XAV-939 as described in Materials \& Methods. Progeny virus was collected at indicated time points and virus titers were determined by plaque assay. The data in (A-D) show one experiment representative of two independent experiments with $n=4(A, C)$ or $n=3(B, D)$ for each experiment. 
A

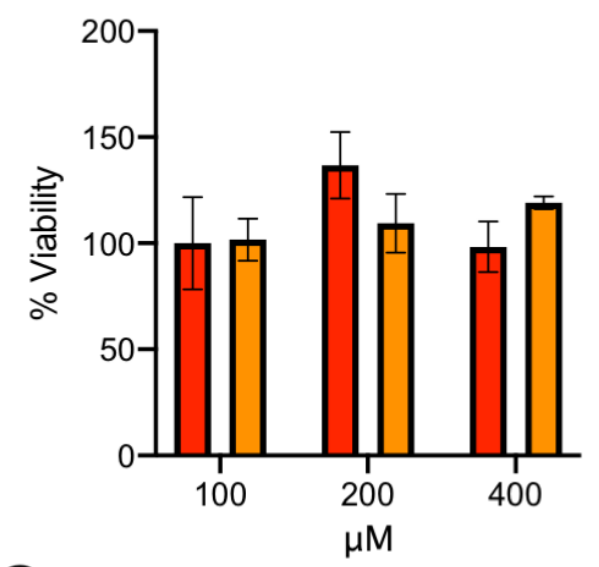

C

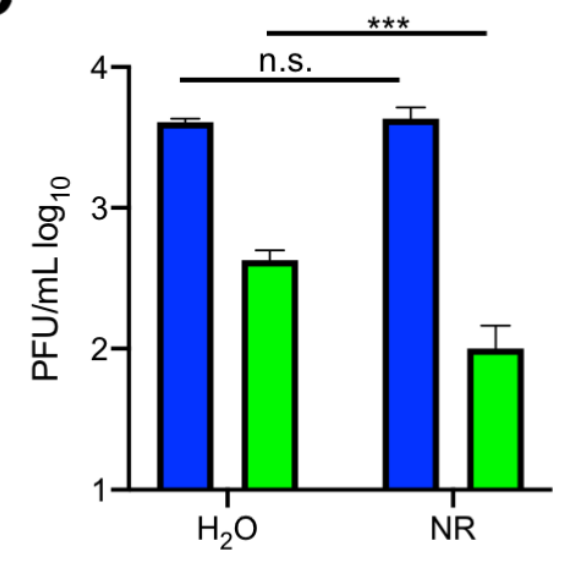

B

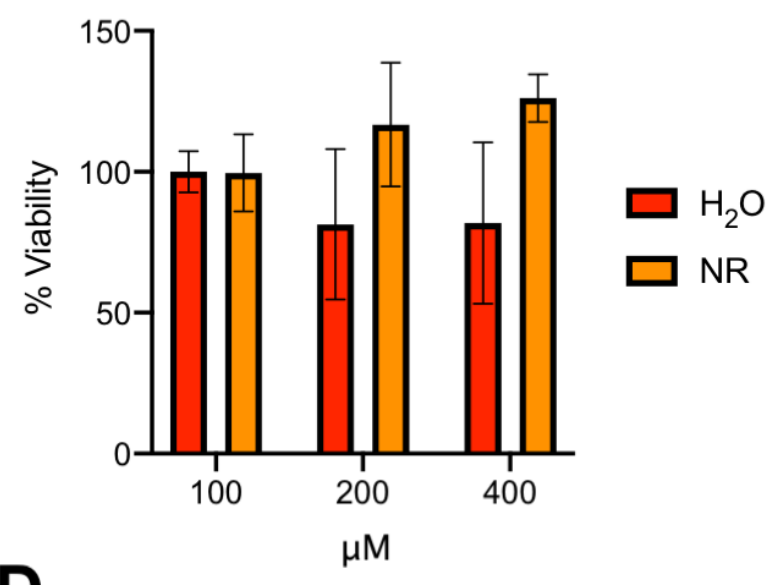

D

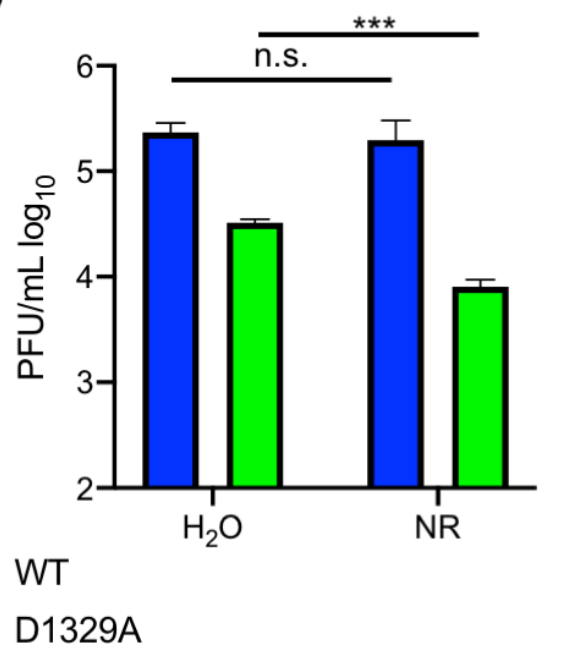

Figure 5. The addition of NR, a precursor of the PARP substrate $\mathrm{NAD}^{+}$, further decreases the replication of D1329A. (A-B) BMDMs (A) and 17Cl-1 cells (B) were treated with NR as described above, and at 24 hours cell viability was measured using an MTT assay as described in Methods. (C-D) WT BMDMs (C) and 17Cl-1 cells (D) were either mock treated $\left(\mathrm{H}_{2} \mathrm{O}\right)$ or treated with NR as described in Methods. Progeny virus was collected at indicated time points and virus titers were determined by plaque assay. The data in (A-D) show one experiment representative of two independent experiments with $n=4(A-B)$ or $n=3(C-D)$ for each experiment. 

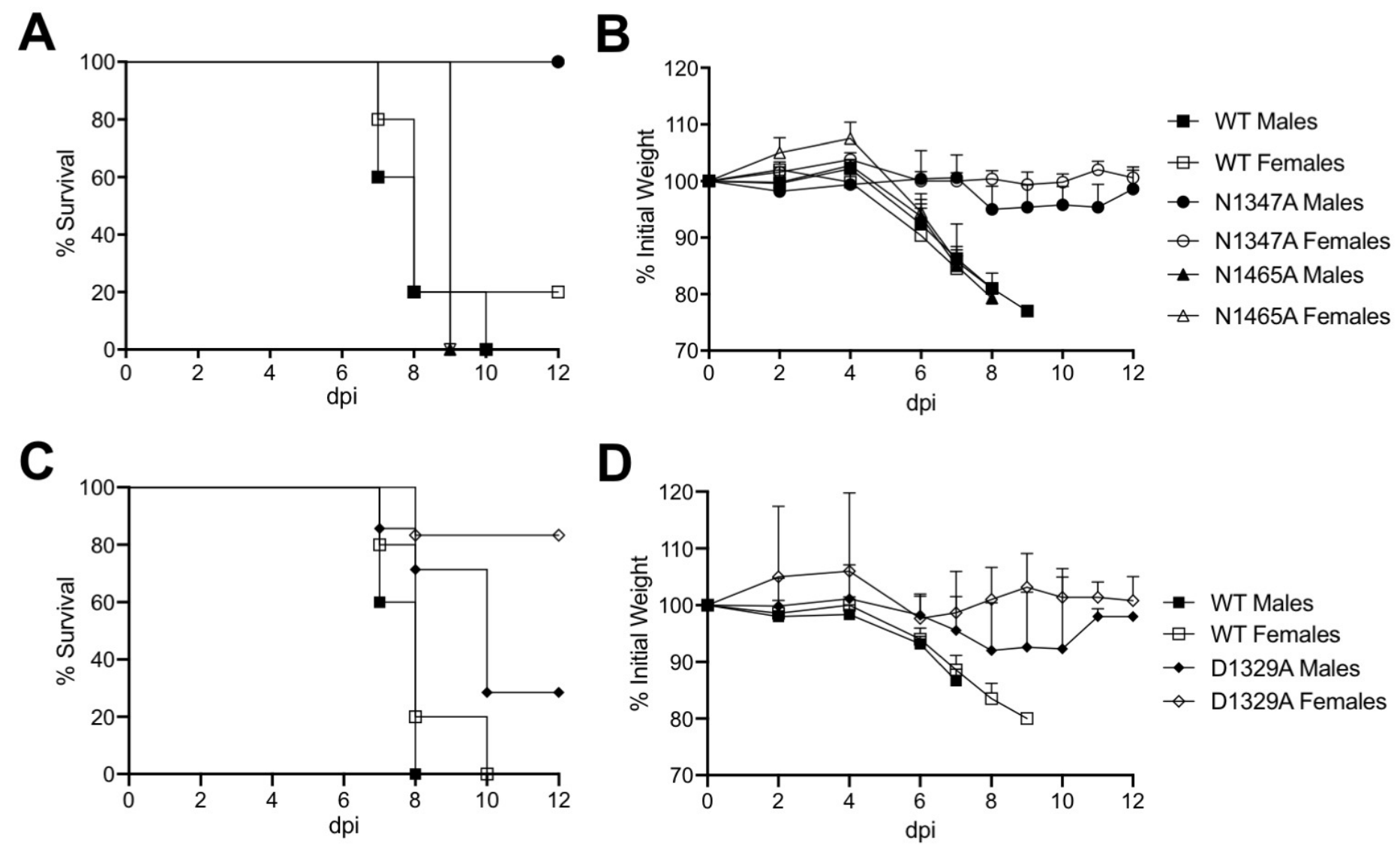

$\mathbf{E}$

\begin{tabular}{llllllllllllllllllll}
$L$ & $Y$ & $G$ & $S$ & $C$ & $I$ & $T$ & $P$ & $N$ & $V$ & $C$ & $F$ & $V$ & $K$ & $G$ & $D$ & $V$ & $I$ & $K$ \\
\hline
\end{tabular}

WT

D1329A TTATATGGTTCATGTATAACACCAAATGTTTGTTTTGTTAAAGGAGCTGTTATAAAG

Mouse \#1 - F ITATATGGTTCATGTATAACACCAAATGTTTGTTTTGTTAAAGGAGATGTTATAAAG A1329D

Mouse \#2 - M ITATATGGTTCATGTATAACACCAAATGTTTGTTTTGTTGAAGGAGCTGTTATAAAG L1313V/K1327E Mouse \#3 - M TTATATGGTTCATGTATAACACCAAATGTTTGTTTTGTTAAAGGAGATGTTATAAAG A1329D

Figure 6. D1329A, but not N1465A, is highly attenuated in vivo. (A-B) WT male and female B6 mice were infected with $3 \times 10^{4} \mathrm{PFU}$ of WT, N1347A, and N1465A intranasally and monitored for survival and weight loss daily for 12 days. The data show the combined results of two independent experiments. WT, $\mathrm{n}=5$ for male and female mice; N1347A, $\mathrm{n}=5$ for male and female mice; N1465A, $\mathrm{n}=4$ for male and female mice. (C-D) WT male and female B6 mice were infected with $3 \times 10^{3} \mathrm{PFU}$ of WT and D1329A as described above. The data show the combined results of two independent experiments. WT, $n=5$ for male and female mice; D1329A, $n=7$ for male and $\mathrm{n}=6$ for female mice. (E) D1329A virus readily reverts in vivo. The brains of 3 mice (2 male (M); 1 female (F)) which succumbed to infection with D1329A were harvested and their viral RNA was amplified by RT-PCR with macrodomain specific primers. The PCR product was sequenced by Sanger sequencing and analyzed using DNA Star software. Mouse \#1 and \#3 reverted to wild-type virus sequence, while mouse \#2 evolved 2 new mutations, L1313V \& K1327E. 

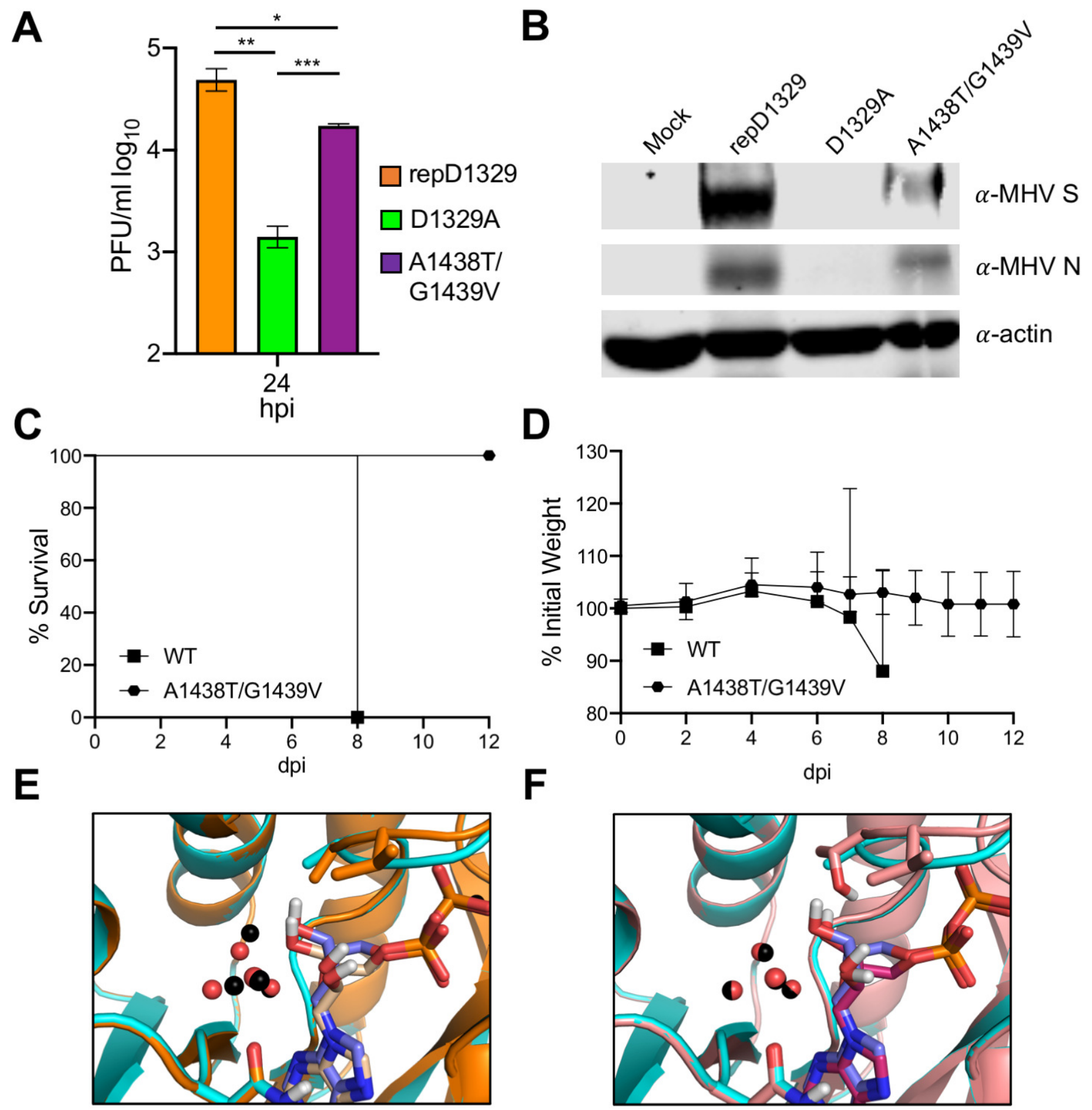

Figure 7. The A1438T/G1439V virus, a revertant of G1439V, is recoverable but replicates at slightly reduced levels compared to WT virus and is attenuated in vivo. (A-B) L929 cells were infected with WT, D1329A, and AG1438/1439TV viruses as described in Materials \& Methods. Progeny virus was collected at 24 hpi and virus titers were determined by plaque assay. In addition cell lysates were collected at 24 hpi and viral protein levels were determined by immunoblotting. The data in (A) shows one experiment representative of two independent experiments with $n=3$ for each experiment. The data in (B) shows one experiment representative of two independent experiments. (C-D) WT male B6 mice were infected with $3 \times 10^{3}$ PFU of WT and A1438T/G1439V intranasally and monitored for survival (C) and weight loss (D) daily for 12 days. WT, n=3; A1438T/G1439V, n=6. (D-E) Rosetta predicted structures of WT MERS-CoV Mac1 around the distal ribose compared to G128V (E) and A127T/G128V (F) proteins. G128V (orange cylinders) is predicted to cause a disruption of water molecules (E) (WT - red spheres; G128V or A127T/G128V - black spheres). The A127T mutation (salmon cylinders) is predicted to restore this water network back to their original location $(\mathrm{F})$, likely restoring critical hydrogen bonds. 